\title{
Integrated, multicohort analysis reveals unified signature of systemic lupus erythematosus
}

\author{
Winston A. Haynes, ${ }^{1,2}$ D. James Haddon, ${ }^{1,3}$ Vivian K. Diep, ${ }^{1,3}$ Avani Khatri, ${ }^{1,3}$ Erika Bongen, ${ }^{1,3}$ \\ Gloria Yiu, ${ }^{1,3}$ Imelda Balboni, ${ }^{4}$ Christopher R. Bolen, ${ }^{1}$ Rong Mao, ${ }^{1,3}$ Paul J. Utz, ${ }^{1,3}$ and Purvesh Khatri ${ }^{1,2}$ \\ ${ }^{1}$ Institute for Immunity, Transplantation and Infection, ${ }^{2}$ Division of Biomedical Informatics Research, ${ }^{3}$ Division of \\ Immunology and Rheumatology, Department of Medicine, and "Division of Allergy, Immunology and Rheumatology, \\ Department of Pediatrics, Stanford University School of Medicine, Stanford, California, USA.
}

\begin{abstract}
Systemic lupus erythematosus (SLE) is a complex autoimmune disease that follows an unpredictable disease course and affects multiple organs and tissues. We performed an integrated, multicohort analysis of 7,471 transcriptomic profiles from 40 independent studies to identify robust gene expression changes associated with SLE. We identified a 93-gene signature (SLE MetaSignature) that is differentially expressed in the blood of patients with SLE compared with healthy volunteers; distinguishes SLE from other autoimmune, inflammatory, and infectious diseases; and persists across diverse tissues and cell types. The SLE MetaSignature correlated significantly with disease activity and other clinical measures of inflammation. We prospectively validated the SLE MetaSignature in an independent cohort of pediatric patients with SLE using a microfluidic quantitative PCR (qPCR) array. We found that 14 of the 93 genes in the SLE MetaSignature were independent of IFN-induced and neutrophil-related transcriptional profiles that have previously been associated with SLE. Pathway analysis revealed dysregulation associated with nucleic acid biosynthesis and immunometabolism in SLE. We further refined a neutropoiesis signature and identified underappreciated transcripts related to immune cells and oxidative stress. In our multicohort, transcriptomic analysis has uncovered underappreciated genes and pathways associated with SLE pathogenesis, with the potential to advance clinical diagnosis, biomarker development, and targeted therapeutics for SLE.
\end{abstract}

Conflict of interest: The authors have declared that no conflict of interest exists.

Copyright: () 2020, American Society for Clinical Investigation.

Submitted: May 17, 2018

Accepted: January 17, 2020

Published: February 27, 2020.

Reference information: /CI Insight. 2020;5(4):e122312.

https://doi.org/10.1172/jici.

insight.122312.

\section{Introduction}

Systemic lupus erythematosus (SLE) is a complex, heterogeneous, chronic autoimmune disease that can affect multiple organs and tissues, including the skin, kidneys, joints, lungs, blood, and CNS. SLE follows an unpredictable disease course, punctuated by periods of flare and remission (1). High-titer, class-switched antibodies that bind to nuclear antigens, including dsDNA, ribonucleoprotein (RNP), Smith, SSA (Ro), and SSB (La), are used in the diagnosis and monitoring of SLE and are thought to be pathogenic. The heterogeneity of SLE makes it challenging for clinicians to manage. Identification of robust molecular changes associated with SLE, despite the patient heterogeneity, will likely improve our understanding and management of SLE.

A number of gene expression studies have shed light on the molecular pathogenesis of SLE. For example, microarray analyses of blood cells derived from patients with SLE have shown that the IFN pathway is dysregulated in a subset of individuals who have more active and severe disease (2-5). Increases in IFN-related genes have also been observed in subsets of patients with other diseases, including systemic sclerosis (SSc), dermatomyositis (DM), polymyositis (PM), primary Sjögren's syndrome (SS), and rheumatoid arthritis (RA), although levels of IFN-inducible gene products were typically highest in SLE (6-10). A review of the biomedical literature identified IFN and neutrophils as major focuses of recent SLE research, with approximately 150 and 40 references per year, respectively. In addition to the IFN signature, upregulation of transcripts associated with granulopoiesis and plasmablasts were observed in individuals who have SLE and were found to be associated with disease activity $(3,5)$. McKinney et al. used gene expression analysis of purified immune cell populations to identify a transcriptional signature in $\mathrm{CD} 8^{+} \mathrm{T}$ cells that was associated with increased likelihood of SLE disease flare (11). They went on to identify an exhaustion 
signature, associated with decreased risk of flare, in $\mathrm{CD}^{+} \mathrm{T}$ cells from individuals who have SLE (12). However, the majority of these studies have been limited by small sample sizes, low levels of clinical and geographic heterogeneity, potential artifacts related to use of a single experimental gene array platform, and lack of external validation. A more robust approach is needed to interrogate the molecular signatures that underlie the highly variable presentation and course of SLE.

We have previously described a multicohort analysis framework (MetaIntegrator) to identify robust disease signatures, and we have repeatedly demonstrated its applications for discovering diagnostics, prognostics and drug targets, and drug repurposing, which leverages the biological and technical heterogeneity present in the large amounts of publicly available gene expression data across a broad spectrum of conditions including infections, organ transplant, vaccination, cancer, and autoimmune diseases (13-15). MetaIntegrator is based on a random-effects meta-analysis, drawing statistical power from the integration of many diverse data sets (14). By computing effect sizes for each data set independently, MetaIntegrator embraces heterogeneity and avoids the limitations of batch effect correction. We have demonstrated application of this framework across a broad spectrum of diseases, including cancer $(16,17)$, solid organ transplant (13), sepsis (18), viral infection (19), tuberculosis (20), neurodegenerative diseases (21), vaccination (22), and SSc (23). Here, we applied the framework to analyze 40 publicly available whole transcriptome profile data sets containing 7471 samples from patients with SLE, individuals with other autoimmune diseases or infections, and healthy volunteers. Together, these data sets represented real-world diversity because of both (a) the biological heterogeneity, as the samples were collected from multiple tissue and cell types (e.g., blood, skin, and kidney) at 17 centers across 5 countries, and (b) the technical heterogeneity, since data were generated using diverse microarray platforms (e.g., Affymetrix arrays, Illumina beadchips, and Hitachisoft chips). Our analysis identified a robust SLE MetaSignature that (a) distinguishes SLE from other autoimmune and inflammatory diseases; (b) is present in multiple affected tissues and immune cell subsets; (c) is independent of age; and (d) is correlated with disease activity. We validated the SLE MetaSignature using additional independent publicly available transcript data sets. We then devised a custom, microfluidic quantitative PCR (qPCR) assay to analyze RNA transcripts in blood derived from a prospective, independent pediatric SLE (pSLE) cohort. Pathway analysis identified potentially novel dysregulated pathways in SLE, including those related to nucleotide biosynthesis and metabolism. Importantly, we identified a non-IFN component of the SLE MetaSignature that correlated more positively with disease activity measures than the IFN-related genes. Finally, our results discovered 14 "non-IFN, nonneutrophil" genes as underappreciated targets for biomarker and therapeutic development.

\section{Results}

Identification of the SLE MetaSignature. To perform a comprehensive, unbiased study of the molecular changes underlying SLE, we identified and downloaded gene expression data from all publicly available human SLE data sets in Gene Expression Omnibus (24). In total, we identified 40 data sets from 17 centers in 5 countries composed of 7471 samples derived from whole blood, peripheral blood mononuclear cells (PBMCs), kidney, skin, synovium, B cells, T cells, monocytes, neutrophils, and endothelial progenitor cells (Figure 1 and Tables 1,2,3). We randomly selected 6 data sets consisting of 370 whole blood and PBMC samples as "Discovery" data sets, based on our previous finding that 5 data sets with 250-300 samples are sufficient to find a robust disease gene signature using our multicohort analysis framework (14). We divided the remaining 34 data sets into "Validation" (2,407 samples in 8 data sets) and "Extended Validation"data sets (4,694 samples in 26 data sets). Discovery and Validation data sets were required to include PBMC or whole blood samples from healthy controls and patients with SLE. Extended Validation data sets included samples from other tissues or cell types, comparisons between SLE and other diseases, and longitudinal SLE samples.

We identified 93 significantly differentially regulated genes (82 upregulated and 11 downregulated) (Supplemental Table 1; supplemental material available online with this article; https://doi.org/10.1172/ jci.insight.122312DS1) with a FDR less than or equal to $5 \%$ and an absolute effect size greater than or equal to 1 compared with healthy volunteers in the Discovery data sets (Figure 2A and Supplemental Table 1). We defined these 93 genes as the "SLE MetaSignature." In the Validation data sets, 73 of these 93 SLE MetaSignature genes met the same filtering criteria ( $|\mathrm{ES}| \geq 1$ and FDR $\leq 5 \%$ ) and effect sizes for all 93 genes exhibited the same directionality as in the Discovery data sets (Figure 2B and Supplemental Figure 1). Of the 20 SLE MetaSignature genes that did not meet the filtering criteria, 18 were statistically significant (FDR $\leq 5 \%$ ) but had an effect size less than 1 (median effect size, 0.78). In the Extended Validation data 


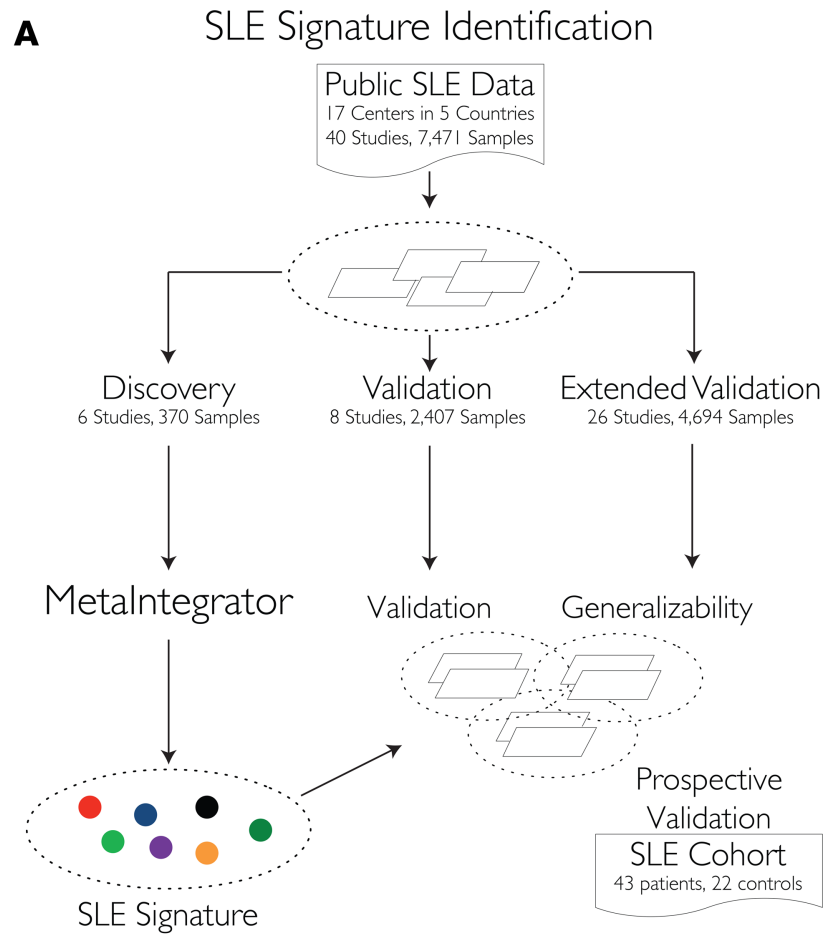

B

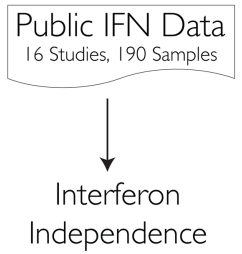

SLE Signature Exploration
Figure 1. Identification and validation of a SLE-specific gene signature using integrated, multicohort analysis. (A) We downloaded 40 publicly available data sets from 17 centers in 5 countries comprising 7,471 samples. We identified data sets that included whole blood or PBMC samples from SLE patients and healthy volunteers to serve as discovery ( 6 studies) and validation (8 studies) sets. The remaining 26 studies contained samples from other tissue types or lacked healthy volunteer samples, and they were examined as extended validation data sets. We used the Metalntegrator framework to identify a 93-gene SLE MetaSignature (effect size $>1, F D R<0.05$, measured in $\geq$ 4 data sets). We examined the classification accuracy of the signature in validation data and the generalizability of the signature in the extended validation data. To prospectively validate the SLE meta-analysis signature using an external cohort, we analyzed individuals who have pSLE $(n=43)$ or JIA $(n=12)$ from the Stanford Pediatric Rheumatology Clinic, as well as healthy adult $(n=10)$ volunteers using Fluidigm qPCR arrays. (B) We leveraged publicly available data to identify non-IFN components of the SLE MetaSignature, examine the role of neutrophils in SLE, and study heavy metal exposure.

sets, which included data from diverse sample types and other diseases, the SLE MetaSignature gene effect sizes were consistent with the Discovery data set (Figure 2C). Regardless of the genetic background of the patients, technical variation, tissue, and cell type, the genes comprising the SLE MetaSignature were all differentially expressed (Figure 2, A-C), demonstrating the robustness of the SLE MetaSignature.

We defined an "SLE MetaScore" for each sample using the 93-gene signature (see Methods). In the Discovery data sets, the SLE MetaScore distinguished SLE patient samples from healthy samples with a summary area under the receiver operating characteristic curve (AUROC) of 0.95 (95\% CI, 0.83-0.99) (Figure 2D). The SLE MetaScore distinguished samples from patients with SLE and healthy volunteers with high accuracy in the 8 Validation data sets (summary AUROC $=0.94 ; 95 \%$ CI, 0.89-0.97) (Figure $2 \mathrm{E})$, further demonstrating the robustness of the SLE MetaSignature.

Of the 93 genes in the SLE MetaSignature, 46 had been previously associated with SLE (2, 3, 5, 25). To the best of our knowledge, the remaining 47 genes have not previously been associated with SLE. We performed pathway analysis of the SLE MetaSignature using Differential Expression Analysis for Pathways (DEAP) (26) to identify biological processes that are dysregulated in SLE. DEAP takes advantage of the meta-analysis effect sizes for all genes (not just those in the SLE MetaSignature) and pathway topology to identify patterns of differential expression that are consistent with known biological pathways. By taking advantage of effect sizes of all genes, DEAP significantly improves power compared with gene list-based approaches (26). Furthermore, DEAP specifies genes involved in the most differentially expressed subpathway. As input for DEAP, we used study level effect sizes from the Discovery and Validation data sets (26). Supplemental Table 2 summarizes pathways that were differentially expressed at a FDR equal to or under $10 \%$ based on 5000 random permutations of the data. In addition to the expected inflammatory pathways (e.g., IFN- $\gamma$ signaling pathway, chemokine/cytokine-mediated 
Table 1. SLE discovery data set summaries

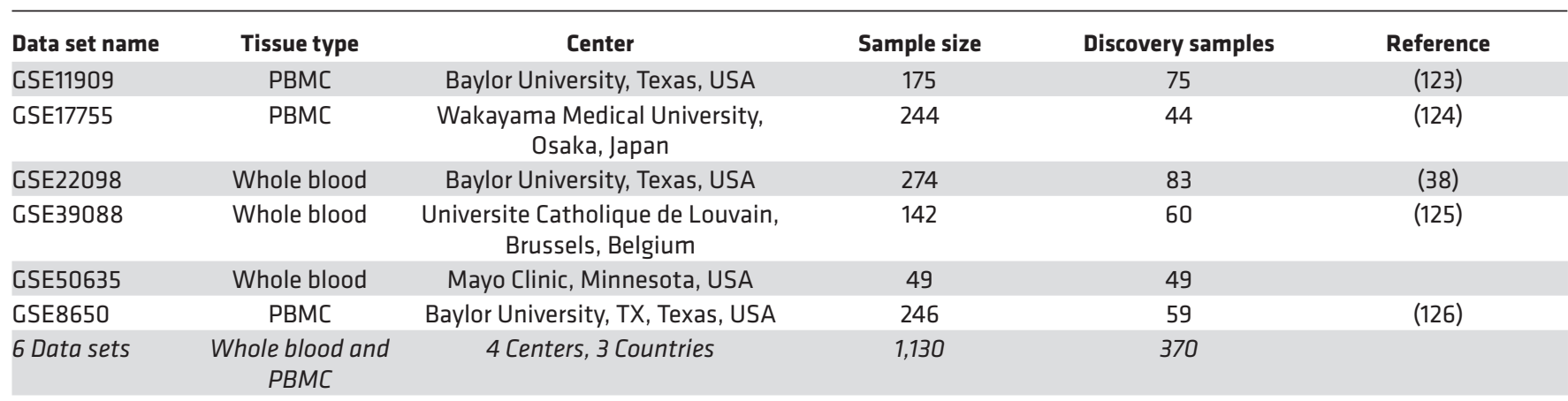

More extensive descriptions in Supplement S1.

signaling pathway, and IL signaling pathway), our analysis identified several highly significant, unexpected pathways (salvage pyrimidine deoxyribonucleotides, formyltetrahydrofolate biosynthesis, and salvage pyrimidine ribonucleotides) related to nucleic acid metabolism. Thus, pathway analysis of the SLE MetaSignature provided insights into the biological mechanisms underlying SLE.

SLE MetaScore distinguishes SLE from other autoimmune, inflammatory, and infectious diseases. We compared SLE MetaScores across inflammatory conditions, including other autoimmune and infectious diseases, to explore its specificity to SLE. We found that adult SLE (aSLE) and patients with pSLE had significantly higher SLE MetaScores than individuals with staphylococcal infection, streptococcal pharyngitis, Still's disease (systemic onset juvenile idiopathic arthritis; sJIA), RA, pyogenic pyoderma gangrenosum and acne (PAPA), B cell deficiency, diabetes, HIV infection, and liver transplant acute rejection in whole blood and PBMC samples across multiple independent data sets (Figure 3, A and B, and Supplemental Figure 2, A and $\mathrm{B})$. In concordance with the previously reported increased severity of disease observed in patients with pSLE compared with adults (27), we found that patients with pSLE had significantly higher SLE MetaScores compared with patients with aSLE (Figure 3B). Taken together, these results demonstrate that, both in adult and pediatric populations, the SLE MetaScore is highly specific to SLE compared with other autoimmune, inflammatory, and infectious diseases.

The SLE MetaScore is systemically higher across tissues in patients with SLE. SLE is a systemic autoimmune disease that affects multiple tissues and organs. Therefore, we explored whether the SLE MetaScore is persistent in tissues other than whole blood and PBMCs in patients with SLE. SLE MetaScores were higher in a data set derived from glomeruli and tubulointerstitium of kidneys from individuals with SLE compared with pretransplant living donors (Figure 3C and Supplemental Figure 3A). SLE MetaScores were higher in a data set from synovial biopsies of patients with SLE compared with those with microcrystalline arthritis

Table 2. SLE validation data set summaries

\begin{tabular}{|c|c|c|c|c|c|}
\hline GSE24706 & PBMC & University of Texas - Southwestern, Texas, USA & 48 & 48 & (128) \\
\hline GSE61635 & PBMC & University of Miami, Florida, USA & 129 & 129 & \\
\hline GSE65391 & Whole blood & Baylor University, TX, Texas, USA & 996 & 230 & (5) \\
\hline GSE72798 & Whole blood & Universite Catholique de Louvain, Brussels, Belgium & 82 & 26 & (130) \\
\hline 8 Data sets & $\begin{array}{l}\text { Whole blood } \\
\text { and PBMC }\end{array}$ & 6 Centers, 3 Countries & 3,196 & 2,407 & \\
\hline
\end{tabular}

More extensive descriptions in Supplement S1. 
Table 3. SLE extended validation data setsummaries

\begin{tabular}{|c|c|c|c|c|}
\hline Data set name & Tissue type & Center & Sample size & Reference \\
\hline GSE24060 & Whole blood & $\begin{array}{l}\text { National Institute of Environmental Health } \\
\text { Sciences, North Carolina, USA }\end{array}$ & 80 & (134) \\
\hline GSE26975 & Neutrophils & University of Michigan, Michigan, USA & 29 & (35) \\
\hline GSE27427 & Neutrophils & Baylor University, Texas, USA & 47 & (136) \\
\hline GSE29536 & Whole blood & Baylor University, Texas, USA & 410 & (137) \\
\hline GSE30153 & B cells & $\begin{array}{c}\text { Institut de Biologie Moléculaire et Cellulaire, } \\
\text { Strasbourg, France }\end{array}$ & 26 & (138) \\
\hline CSE37356 & Sorted cells: monocytes and macrophages & Northwestern University, Illinois, USA & 72 & \\
\hline GSE37573 & B cells: EBV transformed & University of Texas- Southwestern, Texas, USA & 104 & \\
\hline GSE38351 & PBMC & $\begin{array}{c}\text { Deutsches Rheuma-Forschungszentrum, } \\
\text { Berlin, Germany }\end{array}$ & 74 & $(142)$ \\
\hline GSE4588 & Sorted cells: CD4+ T cells, B cells & $\begin{array}{c}\text { Universite Catholique de Louvain, Brussels, } \\
\text { Belgium }\end{array}$ & 49 & \\
\hline GSE46920 & Monocytes & Baylor University, Texas, USA & 12 & (143) \\
\hline GSE46923 & Monocytes & Baylor University, Texas, USA & 142 & (143) \\
\hline GSE50772 & PBMCs & Genentech, California, USA & 81 & $(25)$ \\
\hline GSE51997 & $\begin{array}{l}\text { Sorted cells: CD4 }{ }^{+} \text {T cells, CD16 }{ }^{+} \text {monocytes, } \\
\text { CD16- monocytes }\end{array}$ & $\begin{array}{c}\text { Deutsches Rheuma-Forschungszentrum, } \\
\text { Berlin, Germany }\end{array}$ & 36 & (144) \\
\hline GSE88886 & PBMC & $\begin{array}{l}\text { Eli Lilly and Company, Indianapolis, Indiana, } \\
\text { USA }\end{array}$ & 418 & (131) \\
\hline 26 Data sets & Whole blood, PBMC, sorted cells, tissues & 16 Centers, 4 Countries & 4,056 & \\
\hline
\end{tabular}

More extensive descriptions in Supplement S1.

(gout and pseudogout), osteoarthritis (OA), RA, or seronegative arthritis (Figure 3D). Finally, we found that a data set derived from skin biopsies from individuals with discoid lupus erythematosus exhibited significantly higher SLE MetaScores than healthy volunteers and individuals with psoriasis, suggesting shared pathways between systemic and cutaneous lupus (Supplemental Figure 3B). Collectively, these results provide strong evidence that the SLE MetaScore is higher in multiple affected tissues in SLE in comparison both with healthy controls and other autoimmune diseases.

The SLE MetaScore is differentially expressed in diverse immune cell types. Multiple functional changes have been described in $\mathrm{T}$ cells of patients with SLE, including upregulation of costimulatory molecules, hypomethylation, increased expression of key immune-related genes (28), and aberrant signaling pathway activation downstream of TCR activation (29). We found that the SLE MetaScore was significantly higher in multiple independent data sets from CD4 ${ }^{+} \mathrm{T}$ cells of patients with SLE compared with healthy volunteers (Supplemental Figure 4, A-C) and RA patients (Figure 3E). 
A

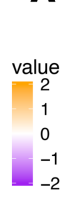

B

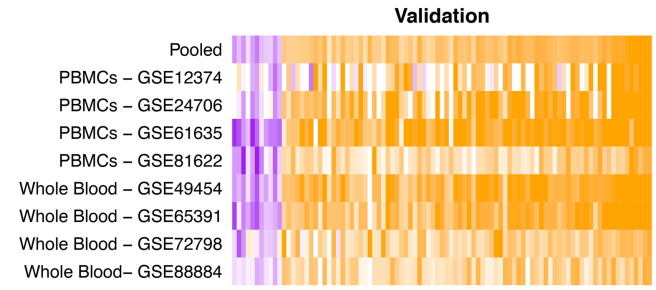

C

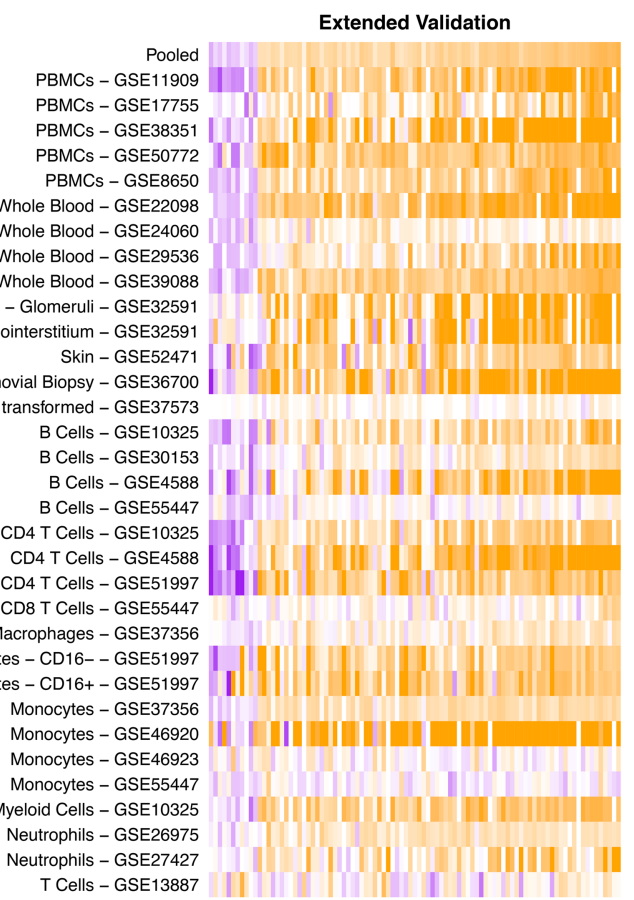
Synovia Bipsy - GSE36700

B Cells - GSE10325

B Cells - GSE30153

B Cells - GSE4588

B Cells - GSE55447

CD4 T Cells - GSE10325

CD4 T Cells - GSE458

CD4 T Cells - GSE51997

CD8 T Cells - GSE55447

Macrophages - GSE3735

Monocytes - CD16- - GSE51997 Monocytes - CD16+ - GSE51997

Monocytes - GSE37356

Monocytes - GSE46920

Monocytes - GSE46923

Monocytes - GSE55447

Myeloid Cells - GSE1032

Neutrophils - GSE26975

Neutrophils - GSE2742

T Cells - GSE1388

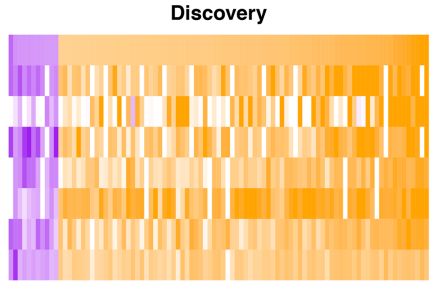

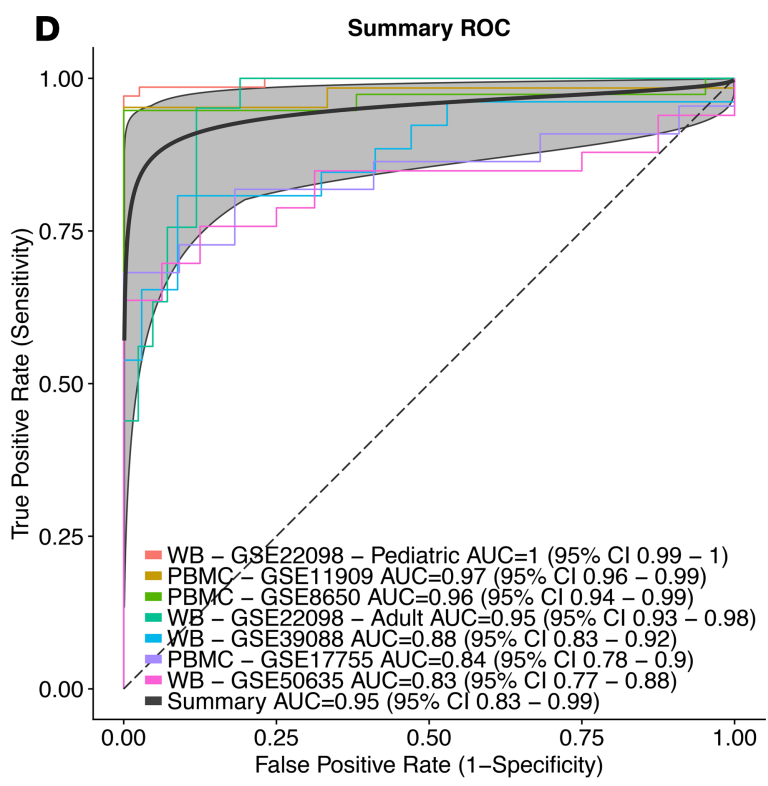

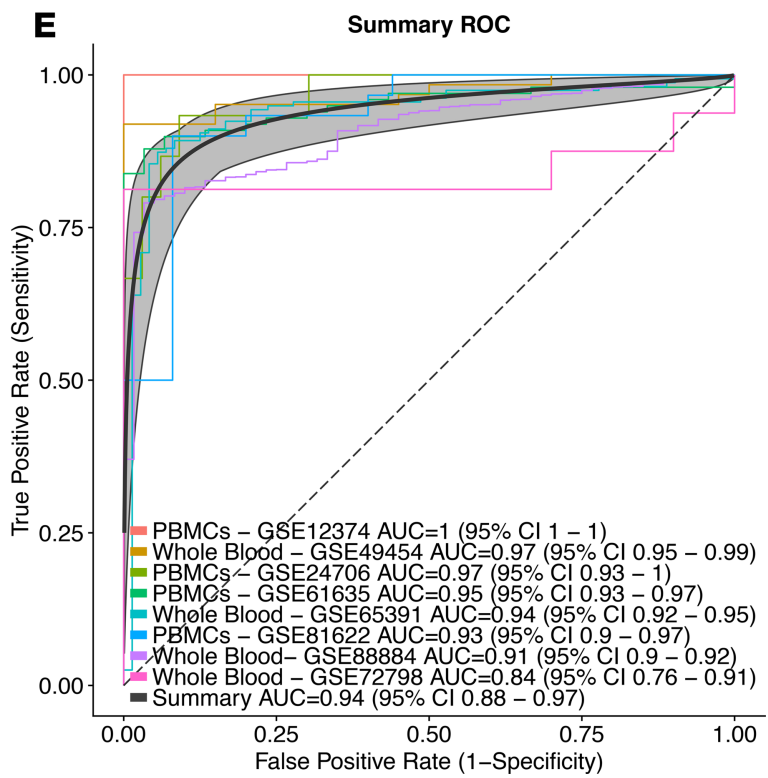

Figure 2. SLE MetaSignature persists across diverse data sets. (A-C) Effect size heatmaps of SLE MetaSignature genes across discovery (A), validation (B), and extended validation (C) data sets. Each column represents a gene in the SLE MetaSignature, ordered from lowest to highest effect size in the discovery data. Each row represents a gene expression data set. ( $\mathbf{D}$ and $\mathbf{E})$ Receiver operating characteristic curves are broken into discovery (D) and validation (E) data. A perfect classifier will have an AUROC of 1 , and a random classifier will have an AUROC of 0.5. We show both whole blood (WB) and peripheral blood mononuclear cell (PBMCs) samples. The summary curve is a composite of the individual study curves. The extended validation ROC plot is shown in Supplemental Figure 9.

Similarly, the SLE MetaScore was significantly increased in a data set from $\mathrm{CD} 8^{+} \mathrm{T}$ cells of individuals with SLE, compared with healthy volunteers (Supplemental Figure 4D).

Dysregulation of B cells is a hallmark of SLE, including autoantibody production, defective negative selection, and changes in the proportions of key B cell subpopulations $(30,31)$. The SLE MetaScore was less robust in data sets from B cells than T cells, classifying SLE in some data sets (Figure 3F and Supplemental Figure 4E) but not others (Supplemental Figure 4, F and G). Finally, the SLE MetaScores in data sets from monocytes and neutrophils were not significantly different between patients with SLE and healthy controls (data not shown).

The SLE MetaScore is positively correlated with disease activity and inflammation. The SLE Disease Activity Index (SLEDAI) is a standardized, albeit imperfect, measure of disease severity and activity. SLEDAI is based on the presence or absence of 24 features at the time of the visit, including arthritis, rash, fever, and increases in antiDNA autoantibodies. It is often used by clinicians to monitor disease activity in an individual SLE patient (3). 

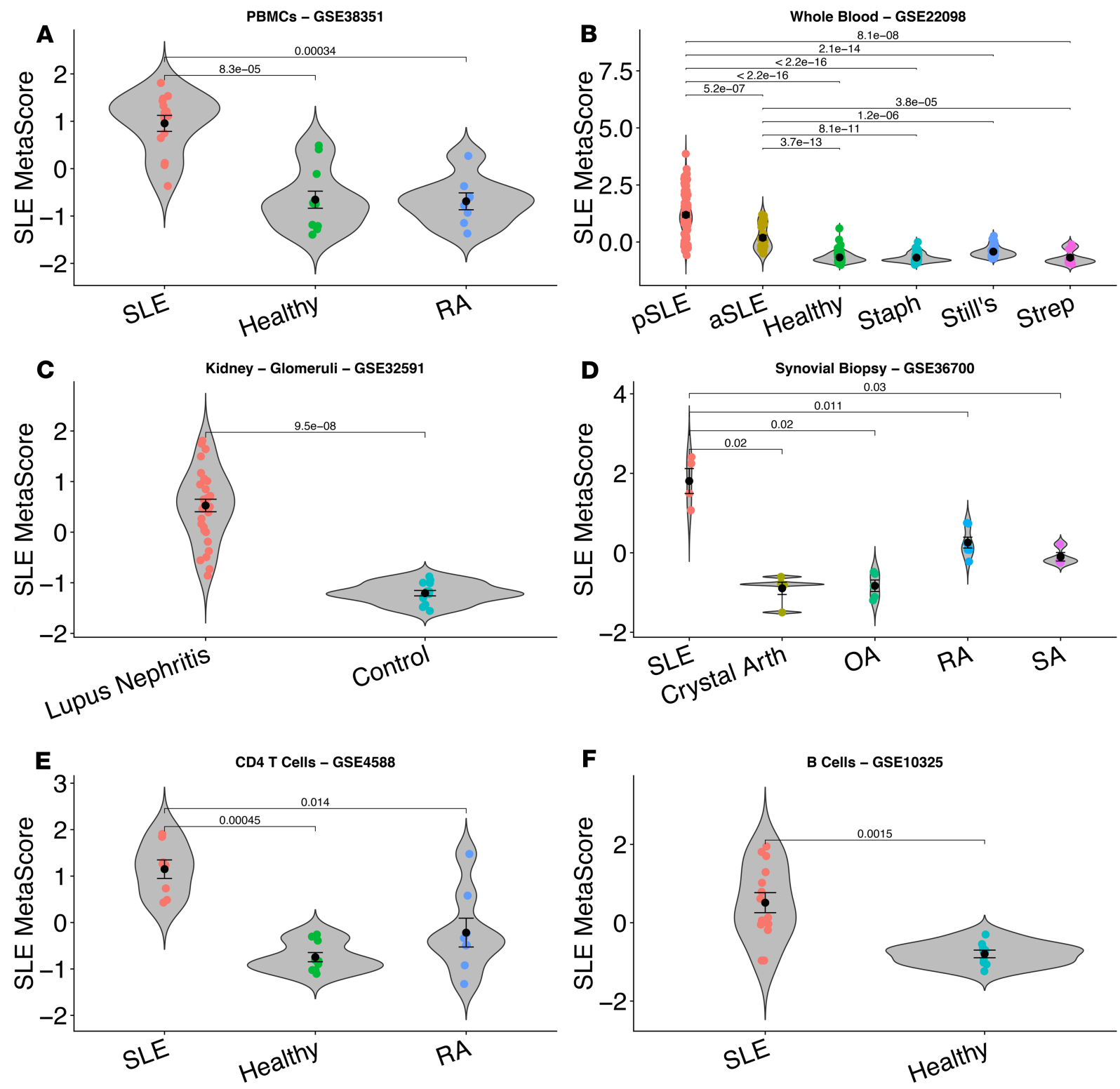

Figure 3. SLE MetaSignature persists across diseases, tissues, and cell types. In these violin plots, each point represents a patient, and the SLE MetaScore ( $y$ axis) has been calculated using the SLE MetaSignature genes. (A and B) The SLE MetaScore distinguished SLE from other diseases. See also Supplemental Figure 2. (C and D) The SLE MetaScore distinguishes SLE from other diseases and healthy controls in diverse tissues. See also Supplemental Figure 3. (E and F) The SLE MetaScore distinguishes SLE patients from healthy and other diseases in sorted immune cells. See also Supplemental Figure 4. RA, rheumatoid arthritis; pSLE, pediatric SLE; aSLE, adult SLE; Staph, staphylococcal infection; Still's, Still's disease; Strep, streptococcal pharyngitis; Crystal Arth, microcrystalline arthritis; OA osteoarthritis; and SA, seronegative arthritis. For all panels, Mann-Whitney $U$ test was used to calculate $P$ values for pairwise comparisons.

Five independent data sets that profiled PBMC or whole blood samples from patients with SLE also reported SLEDAI scores. We observed a positive correlation between SLEDAI and the SLE MetaScore across each of the 5 data sets (Figure 4, A and B, and Supplemental Figure 5, A-D). The median correlation across these studies (correlation of 0.281 ) was significantly elevated compared with random gene sets $(P<0.01)$. The weakest SLEDAI correlation is observed in GSE27427 (Table 3), which contains only 18 samples and is derived from neutrophils. The positive correlation of SLEDAI with SLE MetaScore in the blood is notable, since the SLE MetaSignature was identified without considering disease activity when selecting initial data sets for discovery. Furthermore, we found that the SLE MetaScore correlated highly with individual clinical measures of systemic inflammation, including erythrocyte sedimentation rate (ESR) (Supplemental Figure 5E and ref. 32), and levels of complement C3 (Figure 4C) and C4 (Supplemental Figure 5F). 
A

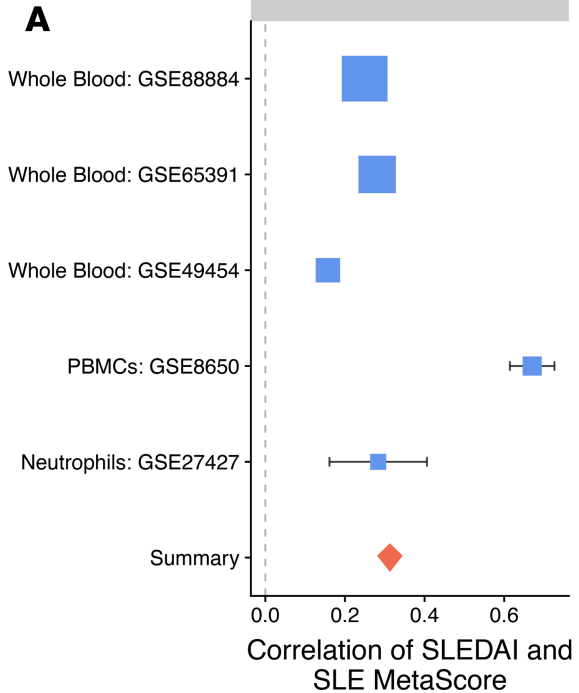

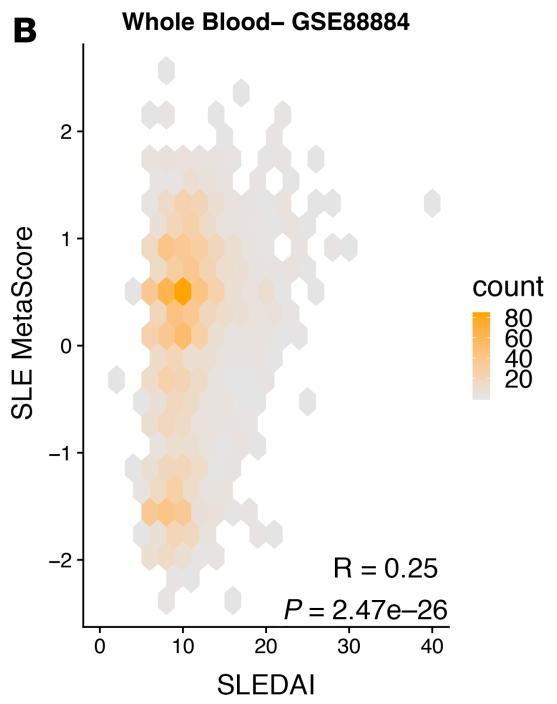

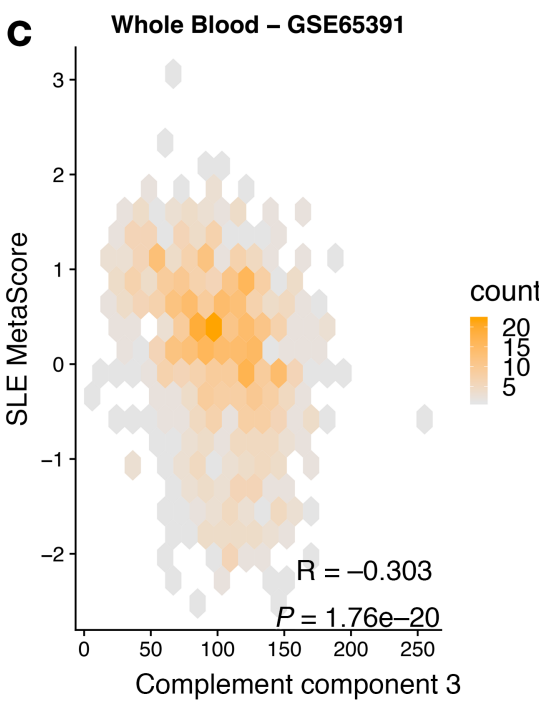

Figure 4. Disease activity is significantly associated with the SLE MetaScore. (A) Positive Spearman correlations are observed across all 5 data sets where SLEDAI was available. Box size is proportional to the confidence of the correlation estimate. Summary is the pooled, inverse variance summary correlation value. (B) The SLE MetaScore in an example SLE whole blood data set is positively correlated with SLEDAI. Spearman's rank correlation $=0.25, P=2.47$ $\times 10^{-26}$ (calculated using AS 89 algorithm; ref. 122). (C) SLE MetaScore is inversely correlated with complement C3 levels. Spearman's rank correlation = $-0.303, P=1.79 \times 10^{-20}$ (calculated using AS 89 algorithm; ref. 122). See also Supplemental Figure 5.

Prospective validation of SLE MetaSignature in an independent pSLE cohort. We validated the SLE MetaSignature in an independent pediatric cohort by studying RNA transcripts in whole blood samples from healthy adult controls and from pediatric patients with SLE or JIA. We selected 33 genes from the SLE MetaSignature based on their significance and availability of validated probes for measuring expression using a microfluidic qPCR array (Supplemental Table 3). Thirty genes out of 33 were significantly differentially expressed in SLE samples compared with healthy adult controls and pediatric JIA patients (FDR $<5 \%$, Supplemental Table 3). Furthermore, the SLE MetaScores based on these 33 genes in the patients with pSLE were significantly higher than healthy adult controls and pediatric JIA patients $\left(P=3.7 \times 10^{-5}\right.$ and $1.8 \times 10^{-6}$, respectively; Figure $\left.5 \mathrm{~A}\right)$; distinguished patients with pSLE with high accuracy (AUROC $=0.94$ ); and were positively correlated with SLEDAI (Spearman's correlation $=0.307, P=0.045$; Figure $5 \mathrm{~B}$ )
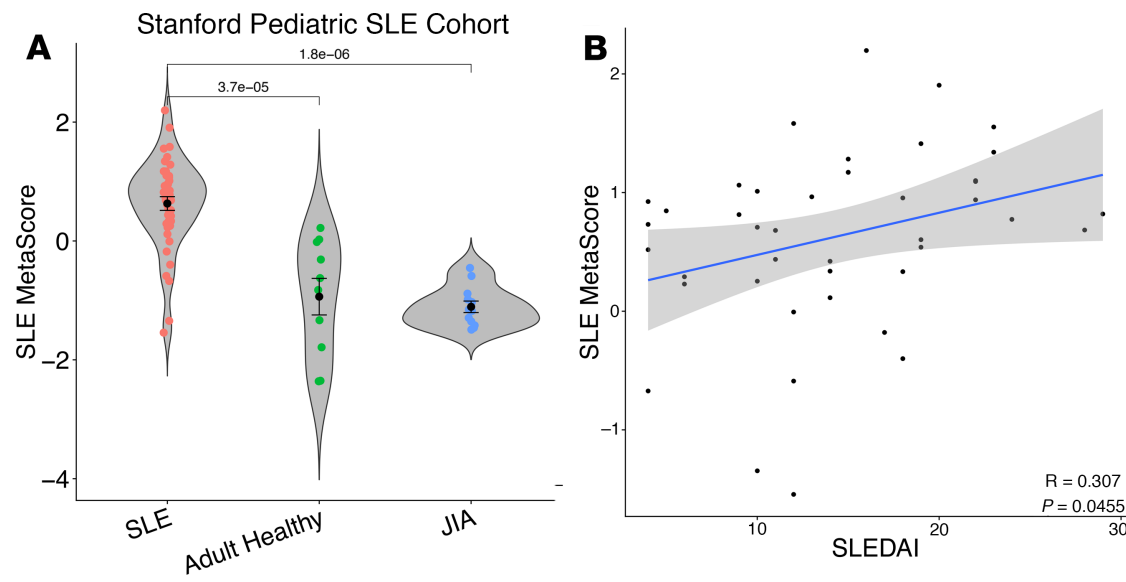

Figure 5. Prospective validation of the SLE MetaSignature in an independent pSLE cohort using a microfluidic qPCR assay. The relative levels of 33 transcripts selected from the SLE MetaSignature (and housekeeping genes) were analyzed in total RNA prepared from the whole blood of new-onset pSLE patients $(n=43)$, individuals with JIA $(n=12)$, and adult healthy volunteers $(n=10)$ in parallel using a multiplexed, microfluidic qPCR assay. (A) The geometric means of the relative concentrations of the 33 transcripts in the SLE MetaSignature were calculated for each individual. Plots show $Z$ scores calculated across individuals. Mann-Whitney $U$ tests were used to compare groups. (B) SLEDAI scores for individual patients were calculated at the time of sampling and were correlated with their SLE MetaScores. Spearman's rank correlation $=0.307, P=0.0455$ (calculated using AS 89 algorithm; ref. 122 ). 


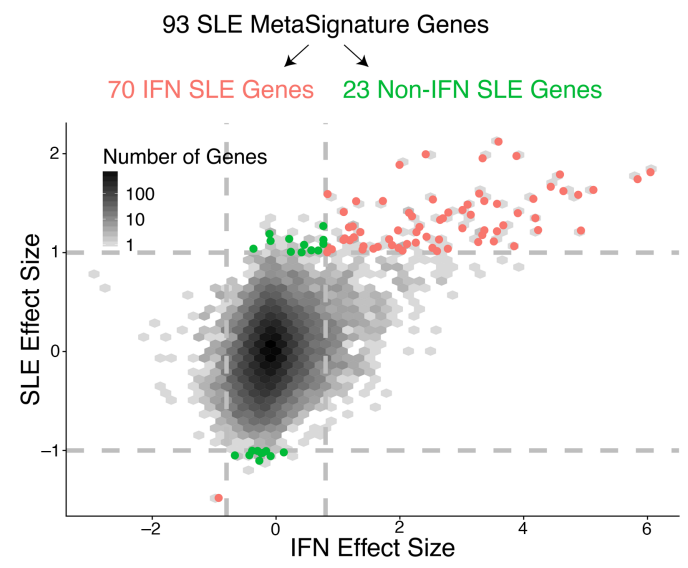

Figure 6. SLE MetaSignature genes dependent or independent of IFN stimulation. Based on our meta-analysis of 16 data sets of type I IFN stimulation in primary cells, we estimated IFN effect sizes in response to stimulation. We compared the SLE MetaSignature to the results of an IFN meta-analysis by examining SLE effect size versus IFN effect size. For a volcano plot for all of the IFN effect sizes, please see Supplemental Figure 6.

A subset of the SLE MetaSignature is not robustly induced by IFN. Dysregulation of the type I IFN pathway has been repeatedly observed in subsets of patients with SLE with active disease and is thought to be a critical mediator in disease pathology. Therefore, we explored the proportion of IFN-stimulated genes in the SLE MetaSignature. We analyzed 16 transcriptome data sets composed of 190 samples derived from primary human cells treated with type I IFN to identify a robust set of type I IFN-stimulated genes (Supplemental Table 4). Of the 93 genes in the SLE MetaSignature, 70 were significantly differentially expressed (effect size $>0.8$ ) in primary cells stimulated by type I IFN (Figure 6 and Supplemental Table 1). The remaining 23 genes in the SLE MetaSignature had low effect sizes and high FDRs within the IFN-stimulated data sets (Supplemental Figure 6), suggesting that these 23 genes were not affected in cells exposed to type I IFN.

We separated the SLE MetaSignature into "IFN" and "non-IFN" SLE MetaSignatures and computed scores as before. Both scores distinguished patients with SLE with equally high accuracy in the validation data sets (Supplemental Table 5). In 4 of 5 data sets with SLEDAI disease severity measurements, the non-IFN SLE MetaSignature had a higher correlation with SLEDAI than the IFN SLE MetaSignature Supplemental Table 6). Collectively, our analyses identified a clinically important, non-IFN component of the SLE MetaSignature.

The role of non-IFN MetaSignature genes in neutrophils. We used immunoStates to identify cell lineages that most highly express genes that comprise the SLE MetaSignature. We found that many of the non-IFN SLE MetaSignature genes were upregulated in neutrophils (33), consistent with prior literature implicating neutrophils in SLE (3, 34-38). Low-density granulocytes exhibit enhanced type I IFN production and NETosis, a form of neutrophil cell death implicated in SLE pathogenesis (39) in which DNA neutrophil extracellular traps (NETs) are extruded from activated neutrophils $(36,39)$. We identified a transcript profiling data set that compared low-density granulocytes and neutrophils from patients with SLE or healthy controls. We observed that the non-IFN SLE MetaSignature was prominently found in low-density granulocytes from patients with SLE but not in neutrophils from patients with SLE or healthy controls (ref. 35 and Figure 7A). We observed a strong correlation between neutrophil abundance and SLE MetaScore in both studies where quantitative neutrophil counts were available (Supplemental Figure 10). Collectively, these results suggest that the SLE MetaSignature genes related to neutrophils are the result of an expansion of the neutrophil compartment in patients with SLE rather than an altered expression profile in SLE neutrophils.

To further explore the role of the non-IFN genes in neutrophils, we identified 4 publicly available gene expression data sets with 84 samples that explored either NETosis or neutrophil development (Supplemental Table 7). The non-IFN SLE MetaSignature was upregulated in cell lines that were stimulated to induce both Nox-dependent and Nox-independent NETosis (Figure 7B). The non-IFN SLE MetaSignature progressively increased during intermediate stages of neutropoiesis (Figure 7C and Supplemental Figure 7, A and B). Collectively, these results indicate that a significant proportion of the non-IFN SLE MetaSignature is related to transcriptional signatures of NETosis and neutropoiesis. 


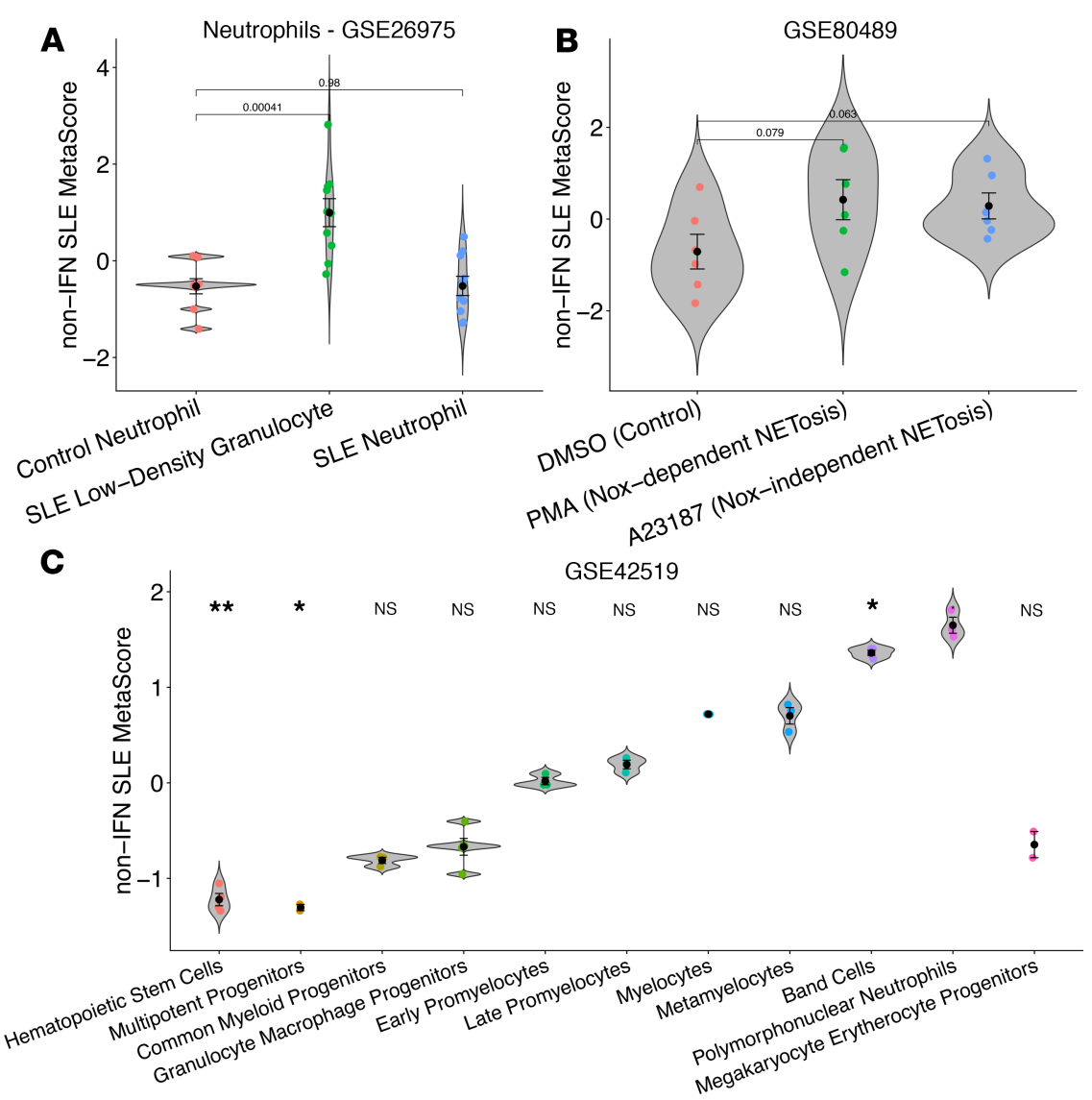

Figure 7. The role of non-IFN MetaSignature genes in SLE neutrophils. We examined the non-IFN component of the SLE MetaSignature (a total of 23 genes) in data sets related to neutrophils. (A) Non-IFN SLE MetaScore in control neutrophils, SLE neutrophils, and SLE low-density granulocytes (LDGs). SLE MetaScore in LDGs is significantly elevated compared with both the control neutrophil and SLE neutrophil populations. (B) Non-IFN SLE MetaScore enriched in primary cells in response to both Nox-dependent (PMA) and Nox-independent (A23187) NETosis. (C) Non-IFN SLE MetaScore progressively increased in sorted intermediate cell populations along the neutropoiesis lineage. See also Supplemental Figure 7. For all panels, Mann-Whitney $U$ test calculated $P$ values, shown for pairwise comparisons. ${ }^{*} P<0.01 ;{ }^{*} P<0.05$.

Identification of underappreciated, non-IFN, nonneutrophil SLE MetaSignature genes. IFN stimulation and neutrophil involvement explained the differential expression of 79 of the 93 genes in the SLE MetaSignature (Figure 8A and Supplemental Table 1). The remaining 14 genes (termed "Underappreciated SLE MetaSignature"; Table 4) provided an opportunity to explore potentially new disease mechanisms that underlie SLE. The underappreciated SLE MetaScore correlated more positively with disease activity measurements than the IFN SLE MetaScore in every blood-derived data set (Supplemental Table 8). Interestingly, 3 members of the metallothionein family (MT1E, MT1F, and MT1HL1) were in the underappreciated SLE MetaSignature. Metallothioneins play an important role in oxidative stress responses and the clearance of heavy metals. We identified 2 data sets in which human cell lines were exposed chronically to cadmium or acutely to zinc. The underappreciated SLE MetaSignature was significantly elevated in cells exposed to heavy metals when compared with the untreated cell lines (ref. 40 and Figure 8, B and C), providing a potential link between SLE and heavy metals, or when exposed to other environmental stimuli that induce oxidative stress. A cadre of the remaining 11 genes in the underappreciated SLE MetaSignature encode molecules with interesting functions related to immune cells, while the remainder of the genes have not been linked to SLE and have yet to be well characterized in the literature.

\section{Discussion}

Previous gene expression meta-analyses in SLE have been limited to a few experiments, lacked external validation, or did not investigate the signature's specificity to SLE $(41,42)$. Our method leverages biological and technical heterogeneity to identify a robust disease signature, and it has been successful in diverse diseases that 
A

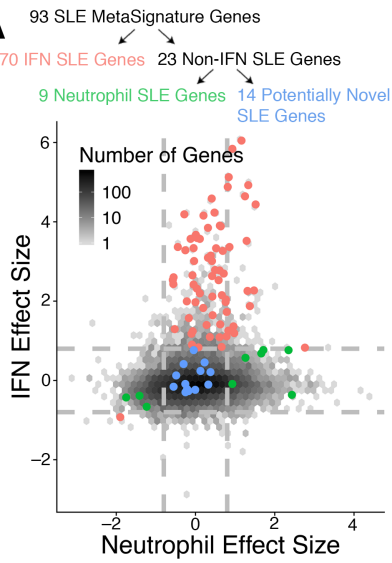

B

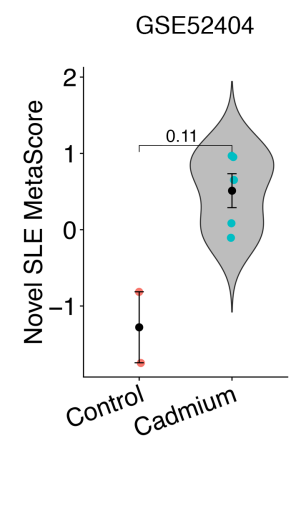

C

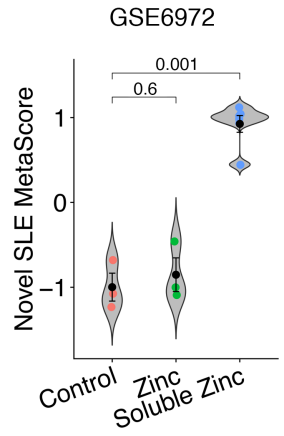

Figure 8. Identification of underappreciated non-IFN, nonneutrophil SLE MetaSignature genes. (A) IFN effect size versus neutrophil effect size. Neutrophil effect size estimated from immunoStates (33). Red indicates the 70 genes that were in the SLE MetaSignature and were significantly differentially expressed in response to IFN. Green indicates the 9 genes that were in the SLE MetaSignature, were not significantly differentially expressed in response to IFN, and were significantly differentially expressed in neutrophils. Blue indicates the 14 genes in the SLE MetaSignature that were not significantly differentially expressed in neutrophils or in response to IFN stimulation. Dashed lines indicate an effect size threshold of $|0.8|$ for both neutrophil and IFN effect sizes. (B) Cell lines that were chronically exposed to cadmium displayed an increased underappreciated SLE MetaScore compared with control cell lines. (C) Cells exposed to a water soluble zinc compound exhibited an increased underappreciated SLE MetaScore compared with those exposed to both a control compound and an insoluble form of zinc (40). For all panels, Mann-Whitney $U$ test was used to calculate $P$ values, shown for pairwise comparisons.

range from cancer to autoimmunity and infection $(13,16-23)$. We performed a multicohort gene expression analysis of more than 7,000 samples from 40 data sets representing real-world biological heterogeneity (including genetic background, age, sex, treatment, tissue, cell type, and disease duration) and technical heterogeneity (including RNA isolation, microarray platform, sample preparation, and experimental protocol) to identify a persistent SLE MetaSignature. The robustness and reproducibility of the SLE MetaSignature demonstrate its generalizability to diverse patient populations not observed in traditional, single-cohort analyses (14).

Beyond generalizability, the SLE MetaSignature was both specific to SLE and correlated with disease activity. Since the SLE MetaSignature distinguished SLE from other diseases, such as diffuse or organ specific autoimmune diseases, inflammatory arthritides, and infectious diseases, the SLE MetaSignature identified SLE-specific disease processes instead of those that are generically dysregulated in other immune-mediated diseases. SLEDAI is the current standard for assessing severity of SLE disease activity, although it is a qualitative, subjective, and difficult-to-reproduce measure (43). Therefore, the positive correlation between the SLE MetaScore and SLEDAI suggests that the SLE MetaScore is not only capturing disease activity, but also is quantitative and objective. Therefore, it could potentially serve as a metric of disease activity in future studies or as an exploratory outcome measure in future clinical trials. Because the SLE MetaScore includes both IFN and non-IFN genes, it expands upon the current best practices of using IFN-focused gene expression to measure quantitative disease activity. Finally, to the best of our knowledge, this is the largest analysis of SLE performed to date that demonstrates that there is a transcriptional signature systemically expressed across different cell types and tissues from patients with SLE and is distinct from other autoimmune and infectious diseases. Our work has the potential to enable more precise molecular definition of SLE that is distinct from other autoimmune diseases.

The role of IFN in SLE has been important in improving the understanding of disease pathogenesis, leading to many publications defining the mechanisms of IFN in SLE (2-5) and several promising clinical trials testing anti-IFN treatments in patients with $\operatorname{SLE}(44,45)$. To explore beyond this existing knowledge about the role of IFN in SLE, we specifically separated the SLE MetaSignature into genes related to IFN and genes that were independent of IFN based on a meta-analysis of 16 transcript profiling data sets from IFN-stimulated human cells. We found that the non-IFN SLE MetaSignature was equally accurate in identifying patients with SLE. Notably, the non-IFN SLE MetaSignature had a higher correlation with SLE disease activity compared with the IFN SLE MetaSignature. Prior studies have likely focused on the IFN-inducible signature due to the high effect sizes of these inflammatory genes. Excluding highly differentially expressed IFN-inducible transcripts allowed us to focus on genes representative of the more nuanced biology underlying SLE. 
Table 4. Underappreciated genes in SLE MetaSignature

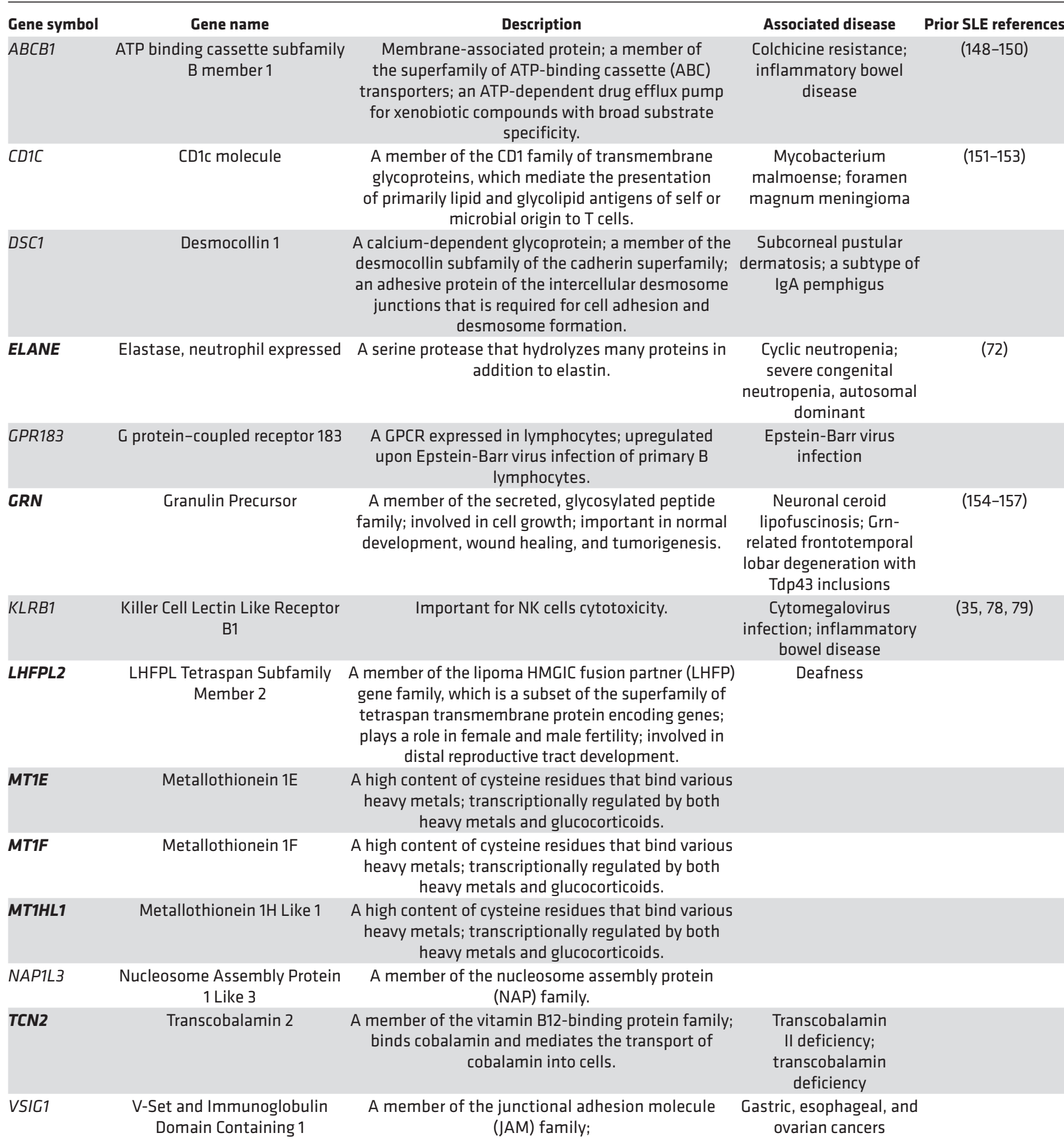

The 14 genes from the underappreciated SLE MetaSignature are listed, including their gene symbol, gene name, description, and human diseases with which these genes have associated. Bolded genes are upregulated in SLE versus healthy controls; downregulated are not bolded.

Neutrophils also play a critical role in SLE pathogenesis. Low-density granulocytes serve as the primary source of proinflammatory NETs (3, 34-39). We found that the non-IFN SLE MetaSignature was most elevated in mature neutrophils, which contrasted with the more immature neutropoiesis signature observed in Bennett et al. (3). The non-IFN SLE MetaSignature was also elevated both in low-density granulocytes and 
in response to NETosis-inducing stimulation. Overall, our work further refines the signature of neutropoiesis in SLE and reinforces an important role for low-density granulocytes and NETosis in SLE. Although B cells have an established role in SLE, the SLE MetaScore exhibited mixed results on available sorted B cell gene expression data sets. Due to limited availability of data, we cannot conclusively evaluate whether these challenges are the result of experimental conditions or a lack of signal in B cells.

One of the most exciting discoveries in the SLE MetaSignature is the identification of 14 genes that are unrelated to type I IFN- or neutrophil-specific gene dysregulation and are genes that, by and large, have not previously been implicated in SLE pathogenesis. These newly identified genes fall into categories that include genes with interesting known biologies that are expressed in immune cells (e.g., $K L R B 1$, GPR183 [also called EBI2], CD1C, and ELANE), genes involved in inflammation and cellular stress responses (MT1E, MT1F, and MT1HL1), and individual genes related to vitamin B12 metabolism (TCN2) and epidermal cellular integrity ( $D S C 1)$.

The most striking group of genes that we identified were members of the metallothionein gene family (MT1E, MT1F, and MT1HL1). Metallothioneins are intracellular, cysteine rich, metal binding proteins involved in diverse intracellular functions that include clearance of heavy metals (cadmium, zinc, and copper) from cells and maintenance of essential ion homeostasis $(46,47)$. Metallothioneins normally bind zinc (48), an important element and potent antioxidant that influences redox state, enzyme activity, gene transcription, energetic metabolism, cell cycle, cell migration, invasivity, apoptosis, and proliferation (49). Both human cell line and animal studies have indicated a role for metallothioneins in protection against cadmium toxicity (50-53). Metallothioneins can be activated by a variety of stimuli, including metal ions, cytokines, and growth factors, as well as oxidative stress and radiation $(49,54)$. During oxidative stress, metallothioneins are upregulated to protect the cells against cytotoxicity, radiation, and DNA damage (5557). Interestingly, metallothionein proteins are expressed at elevated levels in the kidneys of lupus nephritis patients (58). We found that transcript profiles of human cell lines exposed acutely or chronically to heavy metals resembled the underappreciated SLE MetaSignature. We hypothesize that upregulation of metallothioneins in SLE may be a protective response to elevated oxidative stress during chronic inflammatory responses and/or exposure to environmental sources of heavy metals (59-62). The importance of metallothioneins in SLE pathology is underscored by the observation that 2 additional family members ( $M T 1 A$ and $M T 2 A)$ are induced by IFNs and were identified in the 93-gene SLE MetaSignature.

The ELANE gene encodes neutrophil elastase (NE), a serine protease implicated in host defense and tissue injury. In addition to elastin, NE also hydrolyzes proteins within azurophil granules, extracellular matrix proteins, the outer membrane protein A $(\operatorname{Omp} A)$ of E. coli, and the virulence factors of other bacteria (63). In contrast to the digestive serine proteases, NE has unusually high affinity for nucleic acids (64). In naive neutrophils, $\mathrm{NE}$ is normally stored in azurophilic granules $(65,66)$. Upon activation, NE translocates from azurophilic granules to the nucleus, where it partially degrades specific histones, thereby promoting chromatin decondensation and regulating the formation of NETs (67). NE-KO mice are susceptible to bacterial and fungal infections $(68,69)$. Mutations in ELANE can lead to cyclic and severe congenital neutropenia (70). Furthermore, the $\mathrm{NE}$ enzyme may also play a role in various lung, bowel, and skin inflammatory diseases (71). Dysregulation of ELANE in SLE was previously noted in a single-cohort gene expression profile (72). Although known as a neutrophil-expressed gene, we did not identify neutrophil-specific dysregulation of ELANE in our analysis of SLE data sets, and rather than being classified under the neutrophil-related SLE MetaSignature genes, ELANE was classified as an underappreciated SLE MetaSignature gene. Unexpectedly, our further analysis of cell type expression of ELANE using immunoStates (33) indicated that ELANE is most differentially expressed in hematopoietic progenitor cells and basophils (Supplemental Figure 8). This suggests that novel functions for ELANE in other cells, in addition to neutrophils, may be involved in the pathophysiology of SLE.

DSC1 encodes a calcium-dependent glycoprotein in the desmocollin subgroup of the cadherin family. The desmocollins are critical adhesive proteins of the desmosome cell-cell junction linking epithelial cells and are required for cell adhesion and desmosome formation. DSC1 is expressed in the upper epidermis of the skin (73) and has been implicated as an autoantigen for bullous skin disease $(74,75)$, which is also frequently manifested in patients with SLE (75). Mice lacking DSC1 exhibit epidermal fragility accompanied by defects in epidermal barrier and differentiation (76). Neonatal mice lacking desmocollin develop epidermal lesions, and older mice develop ulcerating lesions resembling chronic dermatitis. Based on the above observations, we speculate that the abnormally low levels of DSC1 in patients with SLE lead to reduced adhesion and barrier maintenance of the upper epidermis, increasing the susceptibility to develop bullae and dermatitis. 
KLRB1 (also known as CD161) encodes a C-type lectin-like receptor that is composed of a disulfide-linked homodimer of approximately $40 \mathrm{kDa}$ subunits and is part of the NK gene complex (NKC) (77). $K L R B 1$ has been previously shown to be downregulated in $\operatorname{SLE}(35,78,79)$. This gene is expressed by NK cells, subsets of $\alpha \beta$ and $\gamma \delta \mathrm{T}$ cells, and invariant CD1d-specific NK T cells (80-82). The KLRB1 receptor, by interacting with its ligand $\operatorname{LLT1}(83,84)$, plays an inhibitory role in NK cell-mediated cytotoxicity and IFN- $\gamma$ secretion during immune responses to pathogens $(80,83-85)$. Polymorphisms in KLRB1 are associated with structural alterations of the protein and impact its regulatory functions on NK cell homeostasis and activation (86). In contrast to its inhibitory potential in NK cells, the function of KLRB1 in T cells is less clear, with reports suggesting both coactivating $(81,85,87)$ and inhibitory $(88,89)$ effects. $C D 161$ (KLRB1) has been used as a marker to define Th17 and Tc17 subsets of $\mathrm{CD} 4^{+}$and $\mathrm{CD} 8^{+} \mathrm{T}$ cells that secrete the proinflammatory cytokine IL-17. However, a more recent study found that CD161-expressing $\mathrm{T}$ cell subsets are not all committed to the Th17 axis but are much more diverse, and that expression of CD161 identifies a transcriptional and functional phenotype shared across human $\mathrm{T}$ lymphocytes that is independent of both T cell receptor (TCR) expression and cell lineage (87). The dysregulation of KLRB1 in SLE may be directly linked to aberrant IFN signaling pathways and immune cell subpopulations in this disease.

GPR183 (also known as EBI2) encodes the GPCR183 that binds oxysterols, the most potent of which is $7 \alpha, 25$-dihydroxycholesterol (7 $\alpha, 25-\mathrm{OHC})$ (90). GPR183 is upregulated in a Burkitt's lymphoma cell line upon Epstein-Barr virus infection (91), an infection that is also strongly linked to SLE $(91,92)$. Interesting1y, GPR183 is also strongly induced in UVB-irradiated skin biopsies (93) and UV light has been postulated to induce SLE photosensitivity (94) and DNA damage-driven apoptosis (95). The GPR183 protein is a negative regulator of IFN (96). In lymphoid organs, GPR183 plays a key role in mediating the migration and antibody response of multiple immune cell types, including B cells, T cells, DCs, and monocytes (97-101). GPR183-deficient mice have fewer plasma cells, reduced antibody titres $(97,98)$, and diminished CD4 ${ }^{+}$ splenic DCs. In another study, mice lacking GPR183 or its $7 \alpha, 25-\mathrm{OHC}$ ligand show defects in the trafficking of group 3 innate lymphoid cells and defects in lymphoid tissue formation in the colon (102). GPR183 has been implicated in inflammatory and autoimmune diseases, including multiple sclerosis (103), inflammatory bowel disease (104), Crohn's disease (104), type 1 diabetes, and cancer (101). In multiple sclerosis, data from the experimental autoimmune encephalomyelitis (EAE) animal model suggest that GPR183 is a critical mediator of CNS autoimmunity and regulates the migration of autoreactive T cells into inflamed organs (105). Thus, the intriguing links between GPR183 and SLE through Epstein-Barr virus, IFN, and UV light, as well as its important functions in instructing immune cell localization and antibody response, identify GPR183 and its ligand 7a,25-OHC as potential biomarkers and/or therapeutic targets for SLE.

Research in many immunological disorders, including SLE, has recently focused on the importance of immunometabolism in disease $(106,107)$. In SLE, particular focus has been on T cell metabolism (mitochondria, oxidative stress, mTOR, glucose, and cholesterol pathways), with additional interest in B cells (glycolysis and pyruvate), macrophages (stress response), DCs (mTOR, fatty acids), and neutrophils (NETosis, oxidation) (107). Concordant with these prior findings, our pathway analysis (26) of the SLE MetaSignature recapitulated many similar immunometabolic pathways in SLE, including pyruvate metabolism, fructose galactose metabolism, and oxidative stress response. In addition, our pathway analysis identified many noninflammatory pathways involved in nucleic acid metabolism (including formyltetrahydrofolate biosynthesis, salvage pyrimidine deoxyribonucleotides, salvage pyrimidine ribonucleotides, and purine metabolism). Two therapies in SLE, methotrexate (108) and leflunomide (109-111), both inhibit nucleic acid metabolism (112115), and other new molecular entities that target these pathways are entering clinical trials. Collectively, our pathway analysis results reinforce the importance of immunometabolic pathways in SLE pathogenesis.

Arguably, our approach, which leverages heterogeneity within patient populations to identify a common transcriptional signature across SLE, is ill-suited in the era of personalized medicine. A goal of personalized medicine is to cluster heterogeneous patients into homogeneous subgroups, which does not account for the individual variations that should be targeted. The underlying assumption is that the individual variation between subgroups is likely causal, which can be targeted to improve therapy and outcomes. However, it is equally likely that the disease-causing biology may be the same across all patients, and the variation observed between patients and subgroups is a result of environmental exposures. Studying a homogeneous patient population may identify a signature that explains the variation between groups but may not be causal and therapeutically relevant. Therefore, we believe that a more suitable approach would be to complement "personalized medicine" with "precision medicine" in SLE such that it first provides a precise 
molecular definition of SLE, as we have done here. This could then lead to identification of multiple drug targets and corresponding therapies, increasing the number of drugs available to treat patients with SLE.

We anticipate that the full SLE MetaSignature, and particularly the underappreciated SLE MetaSignature, will be tested in blood and tissue derived from prospectively collected SLE cohorts to identify relationships between SLE flares, clinical subgroups, and responses to newly tested therapies. Another important question is whether the proteins encoded by these genes are abnormally expressed or observed in unanticipated cell populations or tissues. Our results will help guide targeted analyses of SLE blood and kidney samples using single cell technologies such as scRNA-Seq, ATAC-Seq, Cytometry Time of Flight, Multiplexed Ion Beam Imaging, and CO-Detection by IndEXing (116). Many of these methods are being used by the Accelerating Medicines Partnership RA/SLE program to characterize human SLE tissue, with a goal to identify novel pathways and disease targets (117). Ongoing studies using CRISPR screens and IHC are interrogating the role played by these genes in cultured immune cells, as well as the effect of the underappreciated SLE MetaSignature on IFN signaling, neutrophil biology, and animal models.

Our analysis has a few limitations. First, we focused on identifying a gene signature that is conserved between cohorts and across samples and that does not identify patient subgroups. Although this is beneficial for capturing features that are consistent across populations, it is ill-suited for identifying subgroups of disease. Second, because we only used publicly available data sets, our analyses were restricted to the comparisons available in the public data, including tissues, cell types, and diseases sampled. To enable even richer analysis, we encourage the research community to contribute richly annotated data sets to the public domain. In the context of SLE, particularly important annotations - when available — include: age, sex, SLEDAI with individual components specifically recorded, drugs at the time of blood draw, drug doses and start dates, organ system involvement, and cell proportions from complete blood count or flow cytometry.

Recent studies have been dominated by important discoveries that link type I IFN, neutrophils, and NETs to SLE. We have identified a unified SLE MetaSignature that implicates 14 underappreciated genes in SLE pathogenesis, only 4 of which were identified through a direct PubMed search of SLE (KLRB1, GRN, CD1C, and $A B C B 1$, with 2, 5, 7, and 9 references each, respectively). Scouring of published literature reveals connections to additional genes, including ELANE, EBI2, and LHFPL2, but none of these have garnered significant attention in SLE research. Eight of the underappreciated SLE MetaSignature genes have plausible roles in SLE because they are expressed in immune cells, skin, or stress response. Perhaps even more interesting are the 6 genes (ABCB1, GRN, LHFPL2, NAP1L3, TCN2, and VSIG1) that are not linked to the immune system, plausible pathogenic mechanisms, or autoimmune diseases. Scientists often fall prey to the "streetlight effect" - looking for answers where the light is better rather than where the truth is more likely to lie (118-121). Although many of the underappreciated SLE MetaSignature genes make mechanistic sense, we should not lose sight of the 6 genes that had previously been in the shadows but are now illuminated.

\section{Methods}

Supplemental Methods are available online with this article.

Study approval. The vast majority of the data was obtained from public repositories (NCBI GEO). For the prospective validation analysis, all subjects were recruited and all samples were collected following protocols approved by the Stanford University IRB (IRB protocol 13952, 14734).

\section{Author contributions}

WAH, PJU, and PK designed experiments. DJH, VKD, and IB conducted experiments. WAH, EB, and $\mathrm{CRB}$ acquired data. WAH, DJH, EB, and CRB analyzed data. IB and PJU acquired samples. WAH, DJH, $\mathrm{AK}, \mathrm{VKD}, \mathrm{EB}, \mathrm{GY}, \mathrm{IB}, \mathrm{CRB}, \mathrm{RM}, \mathrm{PJU}$, and PK wrote the manuscript.

\section{Acknowledgments}

We would like to thank the pSLE and JIA patients and their families, the healthy volunteers who participated in this study, Human Immune Monitoring Core (HIMC) for performing qPCR assays, and members of the Khatri and Utz laboratories. WAH was funded by the National Science Foundation Graduate Research Fellowship under grant no. DGE-114747 and NIH NLM T15 LM 007033. IB was supported by the NIH (grant no. K08-AI-080945), the Stanford Child Health Research Institute (Child Health Research Program Pilot Grant for Early Career Investigators), and the Arthritis Foundation (Postdoctoral Fellowship). 
EB was supported by the Stanford Gabilan Graduate Fellowship in Science and Engineering and the Stanford Women and Sex Differences in Medicine (WSDM) Seed Grant. GY was supported by the Stanford Medical Scientist Training Program (NIGMS grant no. 5T32GM007365-43). PK is funded by the Bill and Melinda Gates Foundation (OPP1113682); the National Institute of Allergy and Infectious Diseases (NIAID) grants 1U19AI109662, U19AI057229, and 5R01AI125197; Department of Defense contracts W81XWH-18-1-0253 and W81XWH1910235; and the Ralph \& Marian Falk Medical Research Trust. PJU is the recipient of gifts from the Henry Gustav Floren Family Trust, Elizabeth Adler, and the Baxter Foundation. PJU is supported by NIH grants NIAID U19-AI1110491, Stanford Autoimmunity Center of Excellence (ACE); NIAID 1 UM1AI110498-01, ACE Collaborative Project; 1 U19-AI090019; and NIAID 1 R01 AI125197-01.

Address correspondence to: Purvesh Khatri, 279 Campus Drive, Beckman Center, B235A, Stanford, California 94305, USA. Phone: 650.497.5281; Email: pkhatri@stanford.edu. Or to: Paul J. Utz, 269 Campus Drive, CCSR Building, 2215-A, Stanford, California 94305, USA. Phone: 650.724.5421; Email: pjutz@stanford.edu.

1. Tsokos GC. Systemic lupus erythematosus. N Engl J Med. 2011;365(22):2110-2121.

2. Baechler EC, et al. Interferon-inducible gene expression signature in peripheral blood cells of patients with severe lupus. Proc Natl Acad Sci USA. 2003;100(5):2610-2615.

3. Bennett L, et al. Interferon and granulopoiesis signatures in systemic lupus erythematosus blood. J Exp Med. 2003;197(6):711-723.

4. Crow MK, Wohlgemuth J. Microarray analysis of gene expression in lupus. Arthritis Res Ther. 2003;5(6):279-287.

5. Banchereau R, et al. Personalized Immunomonitoring Uncovers Molecular Networks that Stratify Lupus Patients. Cell. 2016;165(3):551-565.

6. Higgs BW, et al. Patients with systemic lupus erythematosus, myositis, rheumatoid arthritis and scleroderma share activation of a common type I interferon pathway. Ann Rheum Dis. 2011;70(11):2029-2036.

7. Emamian ES, et al. Peripheral blood gene expression profiling in Sjögren's syndrome. Genes Immun. 2009;10(4):285-296

8. Baechler EC, et al. An interferon signature in the peripheral blood of dermatomyositis patients is associated with disease activity. Mol Med. 2007;13(1-2):59-68.

9. Greenberg SA, et al. Interferon-alpha/beta-mediated innate immune mechanisms in dermatomyositis. Ann Neurol. 2005;57(5):664-678.

10. Assassi S, et al. Systemic sclerosis and lupus: points in an interferon-mediated continuum. Arthritis Rheum. 2010;62(2):589-598.

11. McKinney EF, et al. A CD8+ T cell transcription signature predicts prognosis in autoimmune disease. Nat Med. 2010;16(5):586-91, 1p following 591.

12. McKinney EF, Lee JC, Jayne DR, Lyons PA, Smith KG. T-cell exhaustion, co-stimulation and clinical outcome in autoimmunity and infection. Nature. 2015;523(7562):612-616.

13. Khatri P, et al. A common rejection module (CRM) for acute rejection across multiple organs identifies novel therapeutics for organ transplantation. J Exp Med. 2013;210(11):2205-2221.

14. Sweeney TE, Haynes WA, Vallania F, Ioannidis JP, Khatri P. Methods to increase reproducibility in differential gene expression via meta-analysis. Nucleic Acids Res. 2017;45(1):e1.

15. Haynes WA, et al. Empowering multi-cohort gene expression analysis to increase reproducibility. Pac Symp Biocomput. 2017;22:144-153.

16. Mazur PK, et al. SMYD3 links lysine methylation of MAP3K2 to Ras-driven cancer. Nature. 2014;510(7504):283-287.

17. Chen R, et al. A meta-analysis of lung cancer gene expression identifies PTK7 as a survival gene in lung adenocarcinoma. Cancer Res. 2014;74(10):2892-2902.

18. Sweeney TE, Shidham A, Wong HR, Khatri P. A comprehensive time-course-based multicohort analysis of sepsis and sterile inflammation reveals a robust diagnostic gene set. Sci Transl Med. 2015;7(287):287ra71.

19. Andres-Terre M, et al. Integrated, Multi-cohort Analysis Identifies Conserved Transcriptional Signatures across Multiple Respiratory Viruses. Immunity. 2015;43(6):1199-1211.

20. Sweeney TE, Braviak L, Tato CM, Khatri P. Genome-wide expression for diagnosis of pulmonary tuberculosis: a multicohort analysis. Lancet Respir Med. 2016;4(3):213-224.

21. Li MD, Burns TC, Morgan AA, Khatri P. Integrated multi-cohort transcriptional meta-analysis of neurodegenerative diseases Acta Neuropathol Commun. 2014;2:93.

22. HIPC-CHI Signatures Project Team, HIPC-I Consortium. Multicohort analysis reveals baseline transcriptional predictors of influenza vaccination responses. Sci Immunol. 2017;2(14):eaal4656.

23. Lofgren S, et al. Integrated, multicohort analysis of systemic sclerosis identifies robust transcriptional signature of disease severity. JCI Insight. 2016;1(21):e89073.

24. Edgar R, Domrachev M, Lash AE. Gene Expression Omnibus: NCBI gene expression and hybridization array data repository. Nucleic Acids Res. 2002;30(1):207-210.

25. Kennedy WP, et al. Association of the interferon signature metric with serological disease manifestations but not global activity scores in multiple cohorts of patients with SLE. Lupus Sci Med. 2015;2(1):e000080.

26. Haynes WA, Higdon R, Stanberry L, Collins D, Kolker E. Differential expression analysis for pathways. PLoS Comput Biol. 2013;9(3):e1002967.

27. Mina R, Brunner HI. Update on differences between childhood-onset and adult-onset systemic lupus erythematosus. Arthritis Res Ther. 2013;15(4):218.

28. Lu Q, Wu A, Tesmer L, Ray D, Yousif N, Richardson B. Demethylation of CD40LG on the inactive X in T cells from women 
with lupus. J Immunol. 2007;179(9):6352-6358.

29. Enyedy EJ, Nambiar MP, Liossis SN, Dennis G, Kammer GM, Tsokos GC. Fc epsilon receptor type I gamma chain replaces the deficient $\mathrm{T}$ cell receptor zeta chain in $\mathrm{T}$ cells of patients with systemic lupus erythematosus. Arthritis Rheum. 2001;44(5):1114-1121.

30. Dörner T, Giesecke C, Lipsky PE. Mechanisms of B cell autoimmunity in SLE. Arthritis Res Ther. 2011;13(5):243.

31. Dörner T, Jacobi AM, Lipsky PE. B cells in autoimmunity. Arthritis Res Ther. 2009;11(5):247.

32. Ho A, Barr SG, Magder LS, Petri M. A decrease in complement is associated with increased renal and hematologic activity in patients with systemic lupus erythematosus. Arthritis Rheum. 2001;44(10):2350-2357.

33. Vallania F, et al. Leveraging heterogeneity across multiple data sets increases accuracy of cell-mixture deconvolution and reduces biological and technical biases [preprint]. https://doi.org/10.1101/206466. Posted on bioRxiv October 20, 2017.

34. Smith CK, Kaplan MJ. The role of neutrophils in the pathogenesis of systemic lupus erythematosus. Curr Opin Rheumatol. 2015;27(5):448-453.

35. Villanueva E, et al. Netting neutrophils induce endothelial damage, infiltrate tissues, and expose immunostimulatory molecules in systemic lupus erythematosus. J Immunol. 2011;187(1):538-552.

36. Kaplan MJ. Neutrophils in the pathogenesis and manifestations of SLE. Nat Rev Rheumatol. 2011;7(12):691-699.

37. Li L, Xia Y, Chen C, Cheng P, Peng C. Neutrophil-lymphocyte ratio in systemic lupus erythematosus disease: a retrospective study. Int J Clin Exp Med. 2015;8(7):11026-11031.

38. Berry MP, et al. An interferon-inducible neutrophil-driven blood transcriptional signature in human tuberculosis. Nature. 2010;466(7309):973-977.

39. Carmona-Rivera C, Kaplan MJ. Low-density granulocytes: a distinct class of neutrophils in systemic autoimmunity. Semin Immunopathol. 2013;35(4):455-463.

40. Magda D, et al. Synthesis and anticancer properties of water-soluble zinc ionophores. Cancer Res. 2008;68(13):5318-5325.

41. Arasappan D, Tong W, Mummaneni P, Fang H, Amur S. Meta-analysis of microarray data using a pathway-based approach identifies a 37-gene expression signature for systemic lupus erythematosus in human peripheral blood mononuclear cells. BMC Med. 2011;9:65.

42. Toro-Domínguez D, Carmona-Sáez P, Alarcón-Riquelme ME. Shared signatures between rheumatoid arthritis, systemic lupus erythematosus and Sjögren's syndrome uncovered through gene expression meta-analysis. Arthritis Res Ther. 2014;16(6):489.

43. Rodríguez-Pintó I, Espinosa G, Cervera R. The problems and pitfalls in systemic lupus erythematosus drug discovery. Expert Opin Drug Discov. 2016;11(6):525-527.

44. Khamashta M, et al. Sifalimumab, an anti-interferon- $\alpha$ monoclonal antibody, in moderate to severe systemic lupus erythematosus: a randomised, double-blind, placebo-controlled study. Ann Rheum Dis. 2016;75(11):1909-1916.

45. Furie R, et al. Anifrolumab, an Anti-Interferon- $\alpha$ Receptor Monoclonal Antibody, in Moderate-to-Severe Systemic Lupus Erythematosus. Arthritis Rheumatol. 2017;69(2):376-386.

46. Klaassen CD, Liu J, Diwan BA. Metallothionein protection of cadmium toxicity. Toxicol Appl Pharmacol. 2009;238(3):215-220.

47. Templeton DM, Cherian MG. Toxicological significance of metallothionein. Meth Enzymol. 1991;205:11-24.

48. Kägi JH. Overview of metallothionein. Meth Enzymol. 1991;205:613-626.

49. Ruttkay-Nedecky B, et al. The role of metallothionein in oxidative stress. Int J Mol Sci. 2013;14(3):6044-6066.

50. Karin M, Cathala G, Nguyen-Huu MC. Expression and regulation of a human metallothionein gene carried on an autonomously replicating shuttle vector. Proc Natl Acad Sci USA. 1983;80(13):4040-4044.

51. Liu Y, Liu J, Iszard MB, Andrews GK, Palmiter RD, Klaassen CD. Transgenic mice that overexpress metallothionein-I are protected from cadmium lethality and hepatotoxicity. Toxicol Appl Pharmacol. 1995;135(2):222-228.

52. Masters BA, Kelly EJ, Quaife CJ, Brinster RL, Palmiter RD. Targeted disruption of metallothionein I and II genes increases sensitivity to cadmium. Proc Natl Acad Sci USA. 1994;91(2):584-588.

53. Enger MD, Tesmer JG, Travis GL, Barham SS. Clonal variation of cadmium response in human tumor cell lines. Am JPhysiol. 1986;250(2 Pt 1):C256-C263.

54. Brüwer M, Schmid KW, Metz KA, Krieglstein CF, Senninger N, Schürmann G. Increased expression of metallothionein in inflammatory bowel disease. Inflamm Res. 2001;50(6):289-293.

55. Sato M, Bremner I. Oxygen free radicals and metallothionein. Free Radic Biol Med. 1993;14(3):325-337.

56. Iszard MB, et al. Characterization of metallothionein-I-transgenic mice. Toxicol Appl Pharmacol. 1995;133(2):305-312.

57. Chubatsu LS, Meneghini R. Metallothionein protects DNA from oxidative damage. Biochem J. 1993;291 ( Pt 1):193-198.

58. Faurschou M, Penkowa M, Andersen CB, Starklint H, Jacobsen S. The renal metallothionein expression profile is altered in human lupus nephritis. Arthritis Res Ther. 2008;10(4):R76.

59. Ashraf MW. Levels of heavy metals in popular cigarette brands and exposure to these metals via smoking. ScientificWorldJournal. 2012;2012:729430.

60. Wolfsperger M, Hauser G, Gössler W, Schlagenhaufen C. Heavy metals in human hair samples from Austria and Italy: influence of sex and smoking habits. Sci Total Environ. 1994;156(3):235-242.

61. Manahan SE. Toxilogical Chemistry and Biochemistry. Boca Raton, Florida, USA: CRC Press; 2002.

62. Huang YC, Li Z, Carter JD, Soukup JM, Schwartz DA, Yang IV. Fine ambient particles induce oxidative stress and metal binding genes in human alveolar macrophages. Am J Respir Cell Mol Biol. 2009;41(5):544-552.

63. Belaaouaj A, Kim KS, Shapiro SD. Degradation of outer membrane protein A in Escherichia coli killing by neutrophil elastase. Science. 2000;289(5482):1185-1188.

64. Thomas MP, et al. Leukocyte protease binding to nucleic acids promotes nuclear localization and cleavage of nucleic acid binding proteins. J Immunol. 2014;192(11):5390-5397.

65. Borregaard N, Cowland JB. Granules of the human neutrophilic polymorphonuclear leukocyte. Blood. 1997;89(10):3503-3521.

66. Lominadze G, Powell DW, Luerman GC, Link AJ, Ward RA, McLeish KR. Proteomic analysis of human neutrophil granules. Mol Cell Proteomics. 2005;4(10):1503-1521.

67. Papayannopoulos V, Metzler KD, Hakkim A, Zychlinsky A. Neutrophil elastase and myeloperoxidase regulate the formation of neutrophil extracellular traps. J Cell Biol. 2010;191(3):677-691. 
68. Belaaouaj A, et al. Mice lacking neutrophil elastase reveal impaired host defense against gram negative bacterial sepsis. Nat Med. 1998;4(5):615-618.

69. Tkalcevic J, Novelli M, Phylactides M, Iredale JP, Segal AW, Roes J. Impaired immunity and enhanced resistance to endotoxin in the absence of neutrophil elastase and cathepsin G. Immunity. 2000;12(2):201-210.

70. Horwitz MS, Corey SJ, Grimes HL, Tidwell T. ELANE mutations in cyclic and severe congenital neutropenia: genetics and pathophysiology. Hematol Oncol Clin North Am. 2013;27(1):19-41, vii.

71. Henriksen PA. The potential of neutrophil elastase inhibitors as anti-inflammatory therapies. Curr Opin Hematol. 2014;21(1):23-28.

72. Rai R, Chauhan SK, Singh VV, Rai M, Rai G. RNA-seq Analysis Reveals Unique Transcriptome Signatures in Systemic Lupus Erythematosus Patients with Distinct Autoantibody Specificities. PLoS ONE. 2016;11(11):e0166312.

73. Cheng X, et al. Assessment of splice variant-specific functions of desmocollin 1 in the skin. Mol Cell Biol. 2004;24(1):154-163.

74. Hashimoto T, et al. Human desmocollin 1 (Dsc1) is an autoantigen for the subcorneal pustular dermatosis type of IgA pemphigus. J Invest Dermatol. 1997;109(2):127-131.

75. Nousari HC, Anhalt GJ. Bullous skin diseases. Curr Opin Immunol. 1995;7(6):844-852.

76. Chidgey M, et al. Mice lacking desmocollin 1 show epidermal fragility accompanied by barrier defects and abnormal differentiation. J Cell Biol. 2001;155(5):821-832.

77. Bartel Y, Bauer B, Steinle A. Modulation of NK cell function by genetically coupled C-type lectin-like receptor/ligand pairs encoded in the human natural killer gene complex. Front Immunol. 2013;4:362.

78. Lin YL, Lin SC. Analysis of the CD161-expressing cell quantities and CD161 expression levels in peripheral blood natural killer and T cells of systemic lupus erythematosus patients. Clin Exp Med. 2017;17(1):101-109.

79. Crow MK, Wohlgemuth J. Microarray analysis of gene expression in lupus. Arthritis Res Ther. 2003;5(6):279-287.

80. Lanier LL, Chang C, Phillips JH. Human NKR-P1A. A disulfide-linked homodimer of the C-type lectin superfamily expressed by a subset of NK and T lymphocytes. J Immunol. 1994;153(6):2417-2428.

81. Exley M, Porcelli S, Furman M, Garcia J, Balk S. CD161 (NKR-P1A) costimulation of CD1d-dependent activation of human T cells expressing invariant V alpha $24 \mathrm{~J}$ alpha Q T cell receptor alpha chains. J Exp Med. 1998;188(5):867-876.

82. Poggi A, et al. Transendothelial migratory pathways of V delta $1+\mathrm{TCR}$ gamma delta + and V delta $2+\mathrm{TCR}$ gamma delta $+\mathrm{T}$ lymphocytes from healthy donors and multiple sclerosis patients: involvement of phosphatidylinositol 3 kinase and calcium calmodulin-dependent kinase II. J Immunol. 2002;168(12):6071-6077.

83. Aldemir H, et al. Cutting edge: lectin-like transcript 1 is a ligand for the CD161 receptor. J Immunol. 2005;175(12):7791-7795.

84. Rosen DB, Bettadapura J, Alsharifi M, Mathew PA, Warren HS, Lanier LL. Cutting edge: lectin-like transcript-1 is a ligand for the inhibitory human NKR-P1A receptor. J Immunol. 2005;175(12):7796-7799.

85. Germain C, et al. Induction of lectin-like transcript 1 (LLT1) protein cell surface expression by pathogens and interferon- $\gamma$ contributes to modulate immune responses. J Biol Chem. 2011;286(44):37964-37975.

86. Rother S, et al. The c.503T >C Polymorphism in the Human KLRB1 Gene Alters Ligand Binding and Inhibitory Potential of CD161 Molecules. PLoS ONE. 2015;10(8):e0135682.

87. Fergusson JR, et al. CD161 defines a transcriptional and functional phenotype across distinct human T cell lineages. Cell Rep. 2014;9(3):1075-1088.

88. Le Bourhis L, et al. MAIT cells detect and efficiently lyse bacterially-infected epithelial cells. PLoS Pathog. 2013;9(10):e1003681

89. Rosen DB, et al. Functional consequences of interactions between human NKR-P1A and its ligand LLT1 expressed on activated dendritic cells and B cells. J Immunol. 2008;180(10):6508-6517.

90. Hannedouche S, et al. Oxysterols direct immune cell migration via EBI2. Nature. 2011;475(7357):524-527.

91. Birkenbach M, Josefsen K, Yalamanchili R, Lenoir G, Kieff E. Epstein-Barr virus-induced genes: first lymphocyte-specific G protein-coupled peptide receptors. J Virol. 1993;67(4):2209-2220.

92. Harley JB, James JA. Epstein-Barr virus infection induces lupus autoimmunity. Bull NYU Hosp Jt Dis. 2006;64(1-2):45-50.

93. Chen W, Zhang J. Potential molecular characteristics in situ in response to repetitive UVB irradiation. Diagn Pathol. 2016;11(1):129

94. Hasan T, et al. Photosensitivity in lupus erythematosus, UV photoprovocation results compared with history of photosensitivity and clinical findings. Br J Dermatol. 1997;136(5):699-705

95. Dunkern TR, Fritz G, Kaina B. Ultraviolet light-induced DNA damage triggers apoptosis in nucleotide excision repair-deficient cells via Bcl-2 decline and caspase-3/-8 activation. Oncogene. 2001;20(42):6026-6038.

96. Chiang EY, Johnston RJ, Grogan JL. EBI2 is a negative regulator of type I interferons in plasmacytoid and myeloid dendritic cells. PLoS ONE. 2013;8(12):e83457.

97. Gatto D, Paus D, Basten A, Mackay CR, Brink R. Guidance of B cells by the orphan G protein-coupled receptor EBI2 shapes humoral immune responses. Immunity. 2009;31(2):259-269.

98. Pereira JP, Kelly LM, Xu Y, Cyster JG. EBI2 mediates B cell segregation between the outer and centre follicle. Nature. 2009;460(7259):1122-1126.

99. Hannedouche S, et al. Oxysterols direct immune cell migration via EBI2. Nature. 2011;475(7357):524-527.

100. Liu C, et al. Oxysterols direct B-cell migration through EBI2. Nature. 2011;475(7357):519-523.

101. Benned-Jensen T, et al. Ligand modulation of the Epstein-Barr virus-induced seven-transmembrane receptor EBI2: identification of a potent and efficacious inverse agonist. J Biol Chem. 2011;286(33):29292-29302.

102. Emgård J, et al. Oxysterol Sensing through the Receptor GPR183 Promotes the Lymphoid-Tissue-Inducing Function of Innate Lymphoid Cells and Colonic Inflammation. Immunity. 2018;48(1):120-132.e8.

103. Chalmin F, et al. Oxysterols regulate encephalitogenic CD4(+) T cell trafficking during central nervous system autoimmunity. J Autoimmun. 2015;56:45-55.

104. Almlöf JC, et al. Novel risk genes for systemic lupus erythematosus predicted by random forest classification. Sci Rep 2017;7(1):6236.

105. Wanke F, et al. EBI2 Is Highly Expressed in Multiple Sclerosis Lesions and Promotes Early CNS Migration of Encephalitogenic CD4 T Cells. Cell Rep. 2017;18(5):1270-1284.

106. O'Neill LA, Kishton RJ, Rathmell J. A guide to immunometabolism for immunologists. Nat Rev Immunol. 2016;16(9):553-565. 
107. Morel L. Immunometabolism in systemic lupus erythematosus. Nat Rev Rheumatol. 2017;13(5):280-290.

108. Sakthiswary R, Suresh E. Methotrexate in systemic lupus erythematosus: a systematic review of its efficacy. Lupus. 2014;23(3):225-235.

109. Tam LS, Li EK, Wong CK, Lam CW, Szeto CC. Double-blind, randomized, placebo-controlled pilot study of leflunomide in systemic lupus erythematosus. Lupus. 2004;13(8):601-604.

110. Tam LS, Li EK, Wong CK, Lam CW, Li WC, Szeto CC. Safety and efficacy of leflunomide in the treatment of lupus nephritis refractory or intolerant to traditional immunosuppressive therapy: an open label trial. Ann Rheum Dis. 2006;65(3):417-418

111. Wang HY, et al. Induction treatment of proliferative lupus nephritis with leflunomide combined with prednisone: a prospective multi-centre observational study. Lupus. 2008;17(7):638-644.

112. Hryniuk WM, Brox LW, Henderson JF, Tamaoki T. Consequences of methotrexate inhibition of purine biosynthesis in L5178Y cells. Cancer Res. 1975;35(6):1427-1432.

113. Skoog L, Nordenskjöld B, Humla S, Hägerström T. Effects of methotrexate on deoxyribonucleotide pools and nucleic acid synthesis in human osteosarcoma cells. Eur J Cancer. 1976;12(10):839-845.

114. Cherwinski HM, et al. The immunosuppressant leflunomide inhibits lymphocyte proliferation by inhibiting pyrimidine biosynthesis. J Pharmacol Exp Ther. 1995;275(2):1043-1049.

115. Zielinski T, Zeitter D, Müller S, Bartlett RR. Leflunomide, a reversible inhibitor of pyrimidine biosynthesis? Inflamm Res. 1995;44 Supp1 2:S207-S208.

116. Cheung P, Khatri P, Utz PJ, Kuo AJ. Single-cell technologies - studying rheumatic diseases one cell at a time. Nat Rev Rheumatol. 2019;15(6):340-354.

117. National Institute of Arthritis and Muskuloskeletal and Skin Diseases (NIAMS). Grants \& Funding: The NIAMS extramural program. Accelerating Medicines Partnership (AMP). http://www.niams.nih.gov/grants-funding/funded-research/ accelerating-medicines.

118. Freedman DH. Why Scientific Studies Are So Often Wrong: The Streetlight Effect. Discover Magazine. http://www.discovermagazine.com/the-sciences/why-scientific-studies-are-so-often-wrong-the-streetlight-effect. Updated December 9, 2010. Accessed January 27, 2020.

119. Battaglia M, Atkinson MA. The streetlight effect in type 1 diabetes. Diabetes. 2015;64(4):1081-1090.

120. Bulgheresi S. Bacterial cell biology outside the streetlight. Environ Microbiol. 2016;18(8):2305-2318.

121. Rodriguez-Esteban R, Jiang X. Differential gene expression in disease: a comparison between high-throughput studies and the literature. BMC Med Genomics. 2017;10(1):59.

122. Best DJ, Roberts DE. Algorithm AS 89: The Upper Tail Probabilities of Spearman's Rho. Journal of the Royal Statistical Society. Series C (Applied Statistics). 1975;24(3):377-379.

123. Chaussabel D, et al. A modular analysis framework for blood genomics studies: application to systemic lupus erythematosus. Immunity. 2008;29(1):150-164.

124. Lee HM, Sugino H, Aoki C, Nishimoto N. Underexpression of mitochondrial-DNA encoded ATP synthesis-related genes and DNA repair genes in systemic lupus erythematosus. Arthritis Res Ther. 2011;13(2):R63.

125. Lauwerys BR, et al. Down-regulation of Interferon Signature in Systemic Lupus Erythematosus Patients by Active Immunization With Interferon $\alpha$-Kinoid. Arthritis Rheum. 2013;65(2):447-456.

126. Allantaz F, et al. Blood leukocyte microarrays to diagnose systemic onset juvenile idiopathic arthritis and follow the response to IL-1 blockade. J Exp Med. 2007;204(9):2131-2144.

127. Lee HM, et al. Interactions among type I and type II interferon, tumor necrosis factor, and beta-estradiol in the regulation of immune response-related gene expressions in systemic lupus erythematosus. Arthritis Res Ther. 2009;11(1):R1.

128. Li QZ, et al. Risk factors for ANA positivity in healthy persons. Arthritis Res Ther. 2011;13(2):R38.

129. Chiche L et al. Modular Transcriptional Repertoire Analyses of Adults With Systemic Lupus Erythematosus Reveal Distinct Type I and Type II Interferon Signatures. Arthritis Rheumatol. 2014;66(6):1583-1595.

130. Ducreux J, et al. Interferon $\alpha$ kinoid induces neutralizing anti-interferon $\alpha$ antibodies that decrease the expression of interferon-induced and B cell activation associated transcripts: analysis of extended follow-up data from the interferon $\alpha$ kinoid phase I/II study. Rheumatology (Oxford). 2016;55(10):1901-1905.

131. Hoffman RW et al. Gene Expression and Pharmacodynamic Changes in 1,760 Systemic Lupus Erythematosus Patients From Two Phase III Trials of BAFF Blockade With Tabalumab. Arthritis Rheumatol. 2017;69(3):643-654.

132. Becker AM, et al. SLE peripheral blood B cell, T cell and myeloid cell transcriptomes display unique profiles and each subset contributes to the interferon signature. PLoS ONE. 2013;8(6):e67003.

133. Fernandez DR, et al. Activation of mammalian target of rapamycin controls the loss of TCRzeta in lupus T cells through HRES-1/Rab4-regulated lysosomal degradation. J Immunol. 2009;182(4):2063-2073.

134. O'Hanlon TP, et al. Gene expression profiles from discordant monozygotic twins suggest that molecular pathways are shared among multiple systemic autoimmune diseases. Arthritis Res Ther. 2011;13(2):R69.

135. Kahlenberg JM, Thacker SG, Berthier CC, Cohen CD, Kretzler M, Kaplan MJ. Inflammasome activation of IL-18 results in endothelial progenitor cell dysfunction in systemic lupus erythematosus. J Immunol. 2011;187(11):6143-6156.

136. Garcia-Romo GS, et al. Netting neutrophils are major inducers of type I IFN production in pediatric systemic lupus erythematosus. Sci Transl Med. 2011;3(73):73ra20.

137. Park J, et al. Interferon signature in the blood in inflammatory common variable immune deficiency. PLoS ONE. 2013;8(9):e74893.

138. Garaud JC, et al. B cell signature during inactive systemic lupus is heterogeneous: toward a biological dissection of lupus. PLoS ONE. 2011;6(8):e23900.

139. Berthier CC, et al. Cross-species transcriptional network analysis defines shared inflammatory responses in murine and human lupus nephritis. J Immunol. 2012;189(2):988-1001.

140. Nzeusseu Toukap A, et al. Identification of distinct gene expression profiles in the synovium of patients with systemic lupus erythematosus. Arthritis Rheum. 2007;56(5):1579-1588.

141. Terrier B, et al. Restoration of regulatory and effector $\mathrm{T}$ cell balance and B cell homeostasis in systemic lupus erythematosus patients through vitamin D supplementation. Arthritis Res Ther. 2012;14(5):R221. 
142. Smiljanovic B, et al. The multifaceted balance of TNF- $\alpha$ and type I/II interferon responses in SLE and RA: how monocytes manage the impact of cytokines. J Mol Med. 2012;90(11):1295-1309.

143. Rodriguez-Pla A, et al. IFN priming is necessary but not sufficient to turn on a migratory dendritic cell program in lupus monocytes. J Immunol. 2014;192(12):5586-5598.

144. Kyogoku C, et al. Cell-specific type I IFN signatures in autoimmunity and viral infection: what makes the difference? PLoS ONE. 2013;8(12):e83776.

145. Jabbari A, et al. Dominant Th1 and minimal Th17 skewing in discoid lupus revealed by transcriptomic comparison with psoriasis. J Invest Dermatol. 2014;134(1):87-95.

146. Sharma S, Jin Z, Rosenzweig E, Rao S, Ko K, Niewold TB. Widely divergent transcriptional patterns between SLE patients of different ancestral backgrounds in sorted immune cell populations. J Autoimmun. 2015;60:51-58.

147. Welcher AA, et al. Blockade of Interferon- $\gamma$ Normalizes Interferon-Regulated Gene Expression and Serum CXCL10 Levels in Patients With Systemic Lupus Erythematosus. Arthritis Rheumatol. 2015;67(10):2713-2722.

148.Tsujimura S, Saito K, Nakayamada S, Nakano K, Tanaka Y. Clinical relevance of the expression of P-glycoprotein on peripheral blood lymphocytes to steroid resistance in patients with systemic lupus erythematosus. Arthritis Rheum. 2005;52(6):1676-1683.

149. Gonzalez TP, Mucenic T, Brenol JC, Xavier RM, Schiengold M, Chies JA. ABCB1 C1236T, G2677T/A and C3435T polymorphisms in systemic lupus erythematosus patients. Braz J Med Biol Res. 2008;41(9):769-772.

150. Ito A, et al. Sex Differences in the Blood Concentration of Tacrolimus in Systemic Lupus Erythematosus and Rheumatoid Arthritis Patients with CYP3A5*3/*3. Biochem Genet. 2017;55(3):268-277.

151. Hänsel A, et al. Human 6-sulfo LacNAc (slan) dendritic cells have molecular and functional features of an important pro-inflammatory cell type in lupus erythematosus. J Autoimmun. 2013;40:1-8.

152. Garaud JC, et al. B cell signature during inactive systemic lupus is heterogeneous: toward a biological dissection of lupus. PLoS ONE. 2011;6(8):e23900.

153. Sieling PA, et al. Human double-negative T cells in systemic lupus erythematosus provide help for IgG and are restricted by CD1c. J Immunol. 2000;165(9):5338-5344.

154. Chen X, Wen Z, Xu W, Xiong S. Granulin exacerbates lupus nephritis via enhancing macrophage M2b polarization. PLoS ONE. 2013;8(6):e65542.

155. Qiu F, et al. Expression level of the growth factor progranulin is related with development of systemic lupus erythematosus. Diagn Pathol. 2013;8:88.

156. Tanaka A, et al. Serum progranulin levels are elevated in patients with systemic lupus erythematosus, reflecting disease activity. Arthritis Res Ther. 2012;14(6):R244.

157. Wu J, Wei L, Wang W, Zhang X, Chen L, Lin C. Diagnostic value of progranulin in patients with lupus nephritis and its correlation with disease activity. Rheumatol Int. 2016;36(6):759-767. 